\title{
STUDIES OF VARIABILITY IN PROTO-PLANETARY NEBULAE. II. LIGHT AND VELOCITY CURVE ANALYSES OF IRAS 22272+5435 AND 22223+4327
}

\author{
Bruce J. HrivnaK ${ }^{1,6}$, WenXian Lu ${ }^{1}$, Julius Sperauskas ${ }^{2}$, Hans VAN Winckel ${ }^{3}$, David BohlendeR ${ }^{4}$, ANd Laimons Začs ${ }^{2,5}$ \\ ${ }^{1}$ Department of Physics and Astronomy, Valparaiso University, Valparaiso, IN 46383, USA; bruce.hrivnak@ valpo.edu, wen.lu@valpo.edu \\ 2 Vilnius University Observatory, Ciurlionio 29 Vilnius 2009, Lithuania; julius.sperauskas@ ff.vu.lt \\ ${ }^{3}$ Instituut voor Sterrenkunde, K. U. Leuven University, B-3001 Leuven (Heverlee), Belgium; Hans.VanWinckel@ ster.kuleuven.be \\ ${ }^{4}$ Dominion Astrophysical Observatory, Herzberg Institute of Astrophysics, National Research Council of Canada, 5071 West Saanich Road, \\ Victoria, BC V9E 2E7, Canada; David.Bohlender@nrc-cnrc.gc.ca \\ ${ }^{5}$ Faculty of Physics and Mathematics, University of Latvia, Raina bulvaris 19, LV-1586 Riga, Latvia; zacs@ latnet.lv \\ Received 2012 December 21; accepted 2013 January 31; published 2013 March 15
}

\begin{abstract}
We have carried out a detailed observational study of the light, color, and velocity variations of two bright, carbon-rich proto-planetary nebulae, IRAS $22223+4327$ and $22272+5435$. The light curves are based upon our observations from 1994 to 2011, together with published data by Arkhipova and collaborators. They each display four significant periods, with primary periods for IRAS $22223+4327$ and $22272+5435$ being 90 and 132 days, respectively. For each of them, the ratio of secondary to primary period is 0.95 , a value much different from that found in Cepheids, but which may be characteristic of post-asymptotic giant branch (AGB) stars. Fewer significant periods are found in the smaller radial velocity data sets, but they agree with those of the light curves. The color curves generally mimic the light curves, with the objects reddest when faintest. A comparison in seasons when there exist contemporaneous light, color, and velocity curves reveals that the light and color curves are in phase, while the radial velocity curves are $\sim 0.25 P$ out of phase with the light curves. Thus they differ from what is seen in Cepheids, in which the radial velocity curve is $0.50 P$ out of phase with the light curve. Comparison of the observed periods and amplitudes with those of post-AGB pulsation models shows poor agreement, especially for the periods, which are much longer than predicted. These observational data, particularly the contemporaneous light, color, and velocity curves, provide an excellent benchmark for new pulsation models of cool stars in the post-AGB, proto-planetary nebula phase.
\end{abstract}

Key words: planetary nebulae: general - stars: AGB and post-AGB - stars: individual (IRAS 22223+4327, IRAS 22272+5435) - stars: oscillations - stars: variables: general

Online-only material: machine-readable tables

\section{INTRODUCTION}

Proto-planetary nebulae (PPNs) represent a transient stage in the evolution of intermediate-mass stars between the asymptotic giant branch (AGB) and the planetary nebula (PN) phases. The identification and study of these objects began in earnest following the successful mission of the Infrared Astronomy Satellite (IRAS) in 1983. Candidate PPNs were selected on the basis of their mid-infrared excesses and follow-up studies have established the PPN nature of a number of these. Their variability has been known since early studies of these objects (Hrivnak 1997).

Light curve studies of individual PPNs have been carried out by Arkhipova and collaborators (e.g., Arkhipova et al. 2000) and by us. Two of us recently published a long-term (14 years) light curve study of 12 carbon-rich PPNs of F-G spectral types (Hrivnak et al. 2010, Paper I). We determined periods for all of them, ranging from $\sim 40-150$ days, and found inverse relationships between the pulsation period and the effective temperature and between the pulsation period and the maximum seasonal variation in brightness.

In this paper, we carry out a more detailed study of two of these carbon-rich PPNs, IRAS $22223+4327$ and 22272+5435, based on extensive light and velocity curve data. In addition to our published light curve data, we include our newer data and data published by others. Similarly, our new radial velocity data

\footnotetext{
${ }^{6}$ Guest investigator, Dominion Astrophysical Observatory, Herzberg Institute of Astrophysics, National Research Council of Canada.
}

are combined with our published data (Začs et al. 2009). These photometric and velocity data are used to carry out a detailed pulsational study of these objects. From these data, we find and compare the periods for the light and velocity variations and also determine the presence of multiple pulsation periods in the data sets. A consistent phase relationship is seen between the light, color, and velocity curves. Attempts are made to use these to determine the radii and luminosities, but these are not successful. Finally, comparisons are made with the predictions of nonlinear, radial pulsation models.

\section{PROGRAM OBJECTS}

Some basic properties of the two program objects are listed in Table 1 . The initial evidence for the PPN nature of IRAS 22272+5435 (HD 235858, BD+54 2787, SAO 34504, V354 Lac) was presented by Hrivnak \& Kwok (1991), whose observations showed a double-peaked spectral energy distribution and a $\mathrm{G}$ supergiant spectral type, and who discovered the presence of $\mathrm{C}_{2}$ and $\mathrm{C}_{3}$ in absorption in the optical spectrum. Similar properties were later found for IRAS 22223+4327 (V448 Lac, DO 41288; Hrivnak 1995).

As noted above, both are carbon-rich and they both have enhanced $s$-process elements due to nucleosynthesis on the AGB (Začs et al. 1995; Van Winckel \& Reyniers 2000; Reddy et al. 2002). As is typical with PPNs, they each show a double-peaked spectral energy distribution, with one peak in the visible/nearinfrared arising from the reddened photosphere and a second one in the mid-infrared arising from cool $(T \sim 150 \mathrm{~K})$ dust. 
Table 1

Program Objects

\begin{tabular}{lcccccccc}
\hline \hline IRAS ID & $\begin{array}{c}V^{\mathrm{a}} \\
(\mathrm{mag})\end{array}$ & $\begin{array}{c}\left(V-R_{C}\right)^{\mathrm{a}, \mathrm{b}} \\
(\mathrm{mag})\end{array}$ & Sp.T. & $\begin{array}{c}T_{\text {eff }} \\
(\mathrm{K})\end{array}$ & $\log g$ & {$[\mathrm{Fe} / \mathrm{H}]$} & $\mathrm{C} / \mathrm{O}$ & Ref. $^{\mathrm{c}}$ \\
\hline $22223+4327$ & 9.8 & 0.54 & G0 Ia & 6500 & 1.0 & -0.3 & 1.2 & 1 \\
$22272+5435$ & 8.6 & 1.03 & G5 Ia & 5750 & 0.5 & -0.8 & 1.6 & 2 \\
\hline
\end{tabular}

Notes.

a Variable.

${ }^{\mathrm{b}}$ Includes circumstellar and interstellar reddening.

c References for the spectroscopic analyses: (1) Van Winckel \& Reyniers 2000; (2) Reddy et al. 2002.

They are among the small number of objects $(\sim 18$ in the Milky Way) found to display the unidentified " $21 \mu \mathrm{m}$ emission feature" (Volk et al. 1999); they also display the " $30 \mu \mathrm{m}$ emission feature" (Volk et al. 2002) and a variety of infrared aromatic features arising in their dusty circumstellar envelope (Hrivnak et al. 2008). The molecular component of their circumstellar envelope is seen in millimeter-line $\mathrm{CO}$ and $\mathrm{HCN}$ emission (Omont et al. 1993) and also in near-infrared $\mathrm{C}_{2}$ and $\mathrm{CN}$ absorption (Bakker et al. 1997). These indicate an envelope expansion of $10-15 \mathrm{~km} \mathrm{~s}^{-1}$. The nebulae appear small in the sky as imaged with the Hubble Space Telescope. Both show a bright star surrounded by a faint reflection nebula, with sizes of 3." $4 \times 2$ 2. 1 (Siodmiak et al. 2008) and 3".5 × 3".5 (Ueta et al. 2000) for IRAS $22223+4327$ and $22272+5435$, respectively.

\section{NEW PHOTOMETRIC OBSERVATIONS}

New differential photometric observations were carried out at the Valparaiso University Observatory (VUO) during the four seasons from 2008-2009 to 2011-2012. (Since our observations in each season are made between May and February, we will hereafter denote them by the earlier of the double years, in which almost all of the observations were made.) These complement the earlier observations carried out at the VUO from 1994 through 2007. The newer data were obtained with a new, largerformat detector, an SBIG 6303 CCD with $2048 \times 3072$ pixels, which was binned $2 \times 2$, with a resulting binned pixel size of 0.70 on the sky. The data were reduced using IRAF, ${ }^{7}$ and aperture photometry was carried out with an aperture of $11^{\prime \prime}$, similar to what was used in Paper I. Three comparison stars $\left(\mathrm{C}_{1}, \mathrm{C}_{2}, \mathrm{C}_{3}\right)$ were used for each object, with one designated as the primary $\left(C_{1}\right)$ and used for the differential photometry measurements. For IRAS 22223+4327, we used the same comparison stars as listed in Paper I. For IRAS 22272+5435, we replaced two of them with brighter stars available with the larger CCD, and we used a new primary comparison star because the previous one appeared to be increasing slightly in brightness by $\sim 0.010$ mag over the four recent years of observation. Standard $B, V, R_{C}$ filters were used, with $R_{C}$ on the Cousins system. Standardized photometry of the program and comparison stars are listed in Table 2 and standardized differential magnitudes are listed in Tables 3 and 4 . The numbers of new observations are as follows: for IRAS 22223+4327-166(B), $173(V), 176\left(R_{C}\right)$, and for IRAS 22272+5435-167 $(B), 170(V), 170\left(R_{C}\right)$. The precision of the data is good, with statistical uncertainties for IRAS $22223+4327$ of $\sigma \leqslant 0.005$ mag and for IRAS $22272+5435$

\footnotetext{
7 IRAF is distributed by the National Optical Astronomical Observatory, operated by the Association for Universities for Research in Astronomy, Inc., under contract with the National Science Foundation.
}

Table 2

Standard Magnitudes of Program and Comparison Stars ${ }^{\mathrm{a}}$

\begin{tabular}{lcrccc}
\hline \hline Object & GSC ID & $V$ & $B-V$ & $V-R_{C}$ & $\mathrm{Run}^{\mathrm{b}}$ \\
\hline IRAS 22223+4327 & $03212-00676$ & 9.88 & 1.00 & 0.54 & 1 \\
& & 9.85 & 0.93 & 0.54 & 2 \\
$\mathrm{C}_{1}$ & $03212-00672$ & 11.08 & 0.54 & 0.31 & $\ldots$ \\
$\mathrm{C}_{2}$ & $03212-00561$ & 11.88 & 0.33 & 0.18 & $\ldots$ \\
$\mathrm{C}_{3}$ & $03212-00499$ & 12.18 & 1.16 & 0.62 & $\ldots$ \\
IRAS 22272+5435 & $03987-01344$ & 8.56 & 2.07 & 1.01 & 1 \\
& & 8.55 & 1.94 & 1.00 & 2 \\
$\mathrm{C}_{1}$ & $03987-02320$ & 11.63 & 0.58 & 0.33 & $\ldots$ \\
$\mathrm{C}_{2}$ & $03987-00944$ & 11.38 & 0.34 & 0.17 & $\ldots$ \\
$\mathrm{C}_{3}$ & $03987-00512$ & 11.16 & 1.11 & 0.60 & $\ldots$ \\
\hline
\end{tabular}

Notes.

${ }^{\text {a }}$ Uncertainties in the observations are as follows $-V: \pm 0.01, B-V: \pm 0.025$, and $V-R_{C}: \pm 0.015 \mathrm{mag}$.

b Observations made at the VUO on (1) 2009 May 20 and (2) 2012 June 26 (UT), and are averaged together for the comparison stars.

of $\sigma \leqslant 0.010 \mathrm{mag}$ for $B$ and $V$ and $\sigma \leqslant 0.014 \mathrm{mag}$ for $R_{C}$. The lower precision for IRAS $22272+5435$ is due to the larger difference in magnitude between it and the comparison star.

\section{RADIAL VELOCITY OBSERVATIONS AND REDUCTIONS}

New radial velocity observations were made from three different observatories in a concerted effort to obtain good time coverage. These are described below, along with some details of the different data sets.

The radial velocity observations for this program were initially made at the Dominion Astrophysical Observatory (DAO) with the $1.2 \mathrm{~m}$ telescope used at the Coudé focus. Observations were made in two observing intervals: 1991 to 1995 (especially 1991 and 1992), and 2007 to 2011. The 1991-1995 DAO data were obtained with the radial velocity spectrometer (RVS; Fletcher et al. 1982), and they are hereafter referred to as DAO-RVS data. This instrument used a physical spectral mask of an F star, based upon Procyon, and included about 340 of the sharpest stellar lines in the wavelength interval from 4000 to $4600 \AA$ A. The DAO radial velocity measurements from 2007 to 2011 were derived from high-resolution CCD spectra in the wavelength interval from 4350 to $4500 \AA$ by cross-correlation with high signal-to-noise $(\mathrm{S} / \mathrm{N})$ spectra of IAU radial velocity standards taken with the same instrument. They are hereafter referred to as DAO-CCD data. Care was taken to ensure that the two sets of measurements are on the same velocity system. The zero point of the much more numerous RVS observations of standard stars has been adjusted to match that of the DAO photographic data (Scarfe et al. 1990; Scarfe 2010). The velocities of the CCD velocity standards were taken to be those derived at the DAO photographically and with the RVS. These two PPNs, with $\mathrm{G}$ spectral types, possess numerous absorption lines that are moderately sharp, and this results in an observational precision of $\sim 0.7 \mathrm{~km} \mathrm{~s}^{-1}$ for the first interval and $\sim 0.5 \mathrm{~km} \mathrm{~s}^{-1}$ for the second.

Radial velocity observations of IRAS $22272+5435$ were initiated by Začs and Sperauskas in 2005 using the CORAVEL spectrometer of Vilnius University (Upgren et al. 2002), and the observations from 2005 to 2007 have been published (Začs et al. 2009). Additional observations of this object have continued and observations of IRAS $22223+4327$ were initiated in 2008 with 
Table 3

Differential Standard Magnitudes for IRAS 22223+4327

\begin{tabular}{lcccrrrr}
\hline \hline HJD-2,400,000 & $\Delta B$ & $\Delta V$ & $\Delta R_{C}$ & HJD-2,400,000 & $\Delta B$ & $\Delta V$ \\
\hline 54654.7279 & $\ldots$ & $\ldots$ & -1.430 & 55142.5341 & -0.792 & -1.215 & -1.427 \\
54662.7959 & -0.777 & -1.204 & -1.426 & 55144.5962 & -0.787 & -1.216 & -1.439 \\
54663.8319 & -0.792 & -1.208 & -1.431 & 55147.6041 & -0.777 & -1.204 & -1.425 \\
54664.7696 & -0.788 & -1.207 & -1.427 & 55167.6577 & -0.823 & -1.242 & -1.467 \\
54665.6989 & -0.787 & -1.193 & -1.411 & 55171.5303 & -0.834 & -1.250 & -1.466 \\
54670.7185 & -0.795 & -1.210 & -1.432 & 55177.5625 & -1.270 & -1.491 \\
54672.7058 & -0.781 & -1.209 & -1.432 & 55210.5381 & -0.799 & -1.213 & -1.428 \\
54689.7864 & -0.817 & -1.247 & -1.470 & 55214.4813 & -0.810 & -1.218 & -1.448 \\
54694.7635 & -0.848 & -1.248 & -1.467 & 55216.5072 & -0.802 & -1.217 & -1.441 \\
54696.7320 & -0.859 & -1.256 & -1.462 & 55228.5020 & -0.813 & -1.232 & -1.449 \\
\hline
\end{tabular}

Note. ${ }^{a}$ The time represents the mid-time of the $V$ observations. The times for the $B$ and $R_{C}$ observations differ from this by approximately +0.0034 and -0.0020 days, respectively.

(This table is available in its entirety in a machine-readable form in the online journal. A portion is shown here for guidance regarding its form and content.)

Table 4

Differential Standard Magnitudes for IRAS 22272+5435

\begin{tabular}{lcccrrrr}
\hline \hline HJD-2,400,000 & $\Delta B$ & $\Delta V$ & $\Delta R_{C}$ & HJD-2,400,000 & $\Delta B$ & $\Delta V$ \\
\hline 54654.7077 & $\ldots$ & $\ldots$ & -3.784 & 55138.6160 & -1.703 & -3.122 & -3.780 \\
54662.7986 & -1.476 & -2.982 & -3.668 & 55141.6836 & -1.687 & -3.116 & -3.799 \\
54663.8366 & -1.497 & -2.973 & -3.657 & 55142.5434 & -1.664 & -3.098 & -3.805 \\
54665.7128 & -1.489 & -2.971 & -3.657 & 55144.6043 & -1.653 & -3.091 & -3.755 \\
54670.7485 & -1.405 & -2.935 & -3.635 & 55147.6091 & -1.623 & -3.058 & -3.742 \\
54672.7231 & -1.384 & -2.909 & -3.595 & 55167.7125 & -1.462 & -2.938 & -3.606 \\
54689.7924 & -1.204 & -2.791 & -3.511 & 55171.5371 & -1.436 & -2.902 & -3.600 \\
54694.7666 & -1.219 & -2.775 & -3.484 & 55182.5854 & -1.430 & -2.891 & -3.575 \\
54696.7369 & -1.246 & -2.780 & -3.504 & 55210.5726 & -1.423 & -2.909 & -3.569 \\
54697.8296 & -1.214 & -2.757 & -3.473 & 55214.4919 & -1.399 & -2.891 & -3.584 \\
\hline
\end{tabular}

Note. ${ }^{\text {a }}$ The time represents the mid-time of the $V$ observations. The times for the $B$ and $R_{C}$ observations differ from this by approximately +0.0025 and -0.0010 days, respectively.

(This table is available in its entirety in a machine-readable form in the online journal. A portion is shown here for guidance regarding its form and content.)

the CORAVEL spectrometer mounted on the $1.65 \mathrm{~m}$ telescope at the Moletai Observatory (Lithuania). Radial velocities were measured using the physical mask containing 1650 slits spread over the spectral interval 3850-6400 $\AA$ and based mainly on the solar spectrum. Some slits are centered on the lines from Arcturus and Procyon spectra also. The precision in the data is $\sim 0.6-0.7 \mathrm{~km} \mathrm{~s}^{-1}$.

Additional radial velocity observations for both objects were also made from 2009 to 2011 with the fiber-fed echelle spectrograph Hermes on the $1.2 \mathrm{~m}$ Flemish Mercator telescope at La Palma (Raskin et al. 2011). The spectra cover the wavelength range 3900-9000 $\AA$ with a spectral resolution of $\lambda / \Delta \lambda \sim$ 85,000 . The data were reduced using a specifically developed instrument pipeline that made use of discrete cross-correlation; the template was based on a $\mathrm{G}$ star and constructed from the line centers and relative depths of the spectral lines. These resultant velocities have a precision of $\sim 0.2 \mathrm{~km} \mathrm{~s}^{-1}$. The velocities are scaled to the IAU system through observations of IAU radial velocity standards (Udry et al. 1999).

For IRAS 22223+4327, we have obtained a total of 178 observations, 34 from the first DAO interval (DAO-RVS), 42 from the second (DAO-CCD), 66 from CORAVEL, and 36 from Hermes. These are listed in Table 5. For IRAS 22272+5435, we have obtained a total of 202 unpublished observations, 33 from the first DAO interval (DAO-RVS), 44 from the second
(DAO-CCD), 79 from CORAVEL, 41 from Hermes, and an additional five observations kindly made for us by R. McClure with the RVS at the DAO between 1988 and 1991 using a $\mathrm{K}$ star mask (DAO-RVS). These are all listed in Table 6. In addition, we have available 72 CORAVEL observations of IRAS $22272+5435$ published by Začs et al. (2009) obtained with the Moletai Observatory telescope and with the $1.5 \mathrm{~m}$ Steward Observatory telescope on Mt. Lemmon. All of the observations have an internal precision of $\leqslant 1.0 \mathrm{~km} \mathrm{~s}^{-1}$.

Special care was called for in combining these different velocity data sets obtained with different methods. While all are recognized as giving precise velocities, we wanted to be careful to investigate and correct for any peculiar systematic effects. It is known, for example, that a systematic offset occurs for CORAVEL data of red stars of late spectral types (Udry et al. 1999). This is discussed in the following two sections.

\section{VARIABILITY STUDY OF IRAS $22272+5435$}

\subsection{Light Curve Study}

Our earlier $V$ and $R_{C}$ light curves of IRAS 22272+5435 from 1994 through 2007 have been described in Paper I. The light varies in a cyclical pattern with the appearance of a changing amplitude. A period of $\sim 130$ days was found from the light and color curves, with a seasonal $V$ amplitude that varies from 
Table 5

Radial Velocity Observations of IRAS 22223+4327

\begin{tabular}{|c|c|c|c|c|c|}
\hline HJD-2,400,000 & $\begin{array}{c}V_{r} \\
\left(\mathrm{~km} \mathrm{~s}^{-1}\right)\end{array}$ & HJD-2,400,000 & $\begin{array}{c}V_{r} \\
\left(\mathrm{~km} \mathrm{~s}^{-1}\right)\end{array}$ & HJD-2,400,000 & $\begin{array}{c}V_{r} \\
\left(\mathrm{~km} \mathrm{~s}^{-1}\right)\end{array}$ \\
\hline \multicolumn{6}{|c|}{ DAO-RVS } \\
\hline 48470.9777 & -42.31 & 48756.9547 & -40.31 & 49243.9289 & -39.99 \\
\hline 48471.8874 & -42.55 & 48779.9297 & -38.43 & 49263.8801 & -44.54 \\
\hline 48510.8859 & -36.12 & 48788.8747 & -39.39 & 49286.8661 & -41.64 \\
\hline 48511.8290 & -36.13 & 48799.9333 & -42.26 & 49286.8995 & -42.86 \\
\hline 48532.7969 & -38.70 & 48834.8871 & -40.56 & 49554.9029 & -40.90 \\
\hline 48533.8200 & -38.41 & 48837.8878 & -40.06 & 49555.9399 & -41.16 \\
\hline 48551.7917 & -44.11 & 48871.8468 & -40.45 & 49899.9354 & -41.34 \\
\hline 48566.7475 & -43.78 & 48875.8244 & -42.80 & 49913.9602 & -39.74 \\
\hline 48588.7468 & -39.22 & 49130.9690 & -38.28 & 49940.8221 & -38.38 \\
\hline 48627.6548 & -42.38 & 49163.9382 & -41.86 & 50020.6870 & -39.59 \\
\hline 48719.9993 & -39.90 & 49217.9829 & -38.34 & $\ldots$ & $\ldots$ \\
\hline 48733.9357 & -42.56 & 49239.8757 & -38.47 & $\ldots$ & $\ldots$ \\
\hline \multicolumn{6}{|c|}{ DAO-CCD } \\
\hline 54327.8994 & -39.60 & 54790.6756 & -43.65 & 55392.9142 & -42.99 \\
\hline 54338.8103 & -40.28 & 54796.6355 & -43.22 & 55406.9462 & -44.18 \\
\hline 54354.8812 & -42.82 & 54944.9767 & -46.21 & 55426.9406 & -42.63 \\
\hline 54384.7518 & -41.47 & 55014.8890 & -42.00 & 55432.9379 & -41.07 \\
\hline 54391.7458 & -41.51 & 55028.8741 & -41.34 & 55476.8042 & -44.93 \\
\hline 54403.7580 & -42.17 & 55041.8754 & -40.92 & 55482.8715 & -44.12 \\
\hline 54447.7227 & -40.96 & 55050.9065 & -41.64 & 55524.7324 & -40.03 \\
\hline 54658.9294 & -40.59 & 55055.8915 & -42.16 & 55719.9204 & -36.62 \\
\hline 54690.8693 & -39.48 & 55063.8680 & -42.26 & 55812.9217 & -41.79 \\
\hline 54709.8763 & -39.27 & 55076.8689 & -39.74 & 55880.6686 & -37.76 \\
\hline 54719.9476 & -41.87 & 55096.8159 & -39.54 & 55908.7026 & -41.69 \\
\hline 54725.9390 & -42.64 & 55103.8066 & -42.22 & 55916.6871 & -42.10 \\
\hline 54740.9688 & -43.14 & 55167.7556 & -39.45 & 55938.6286 & -45.77 \\
\hline 54754.7945 & -40.50 & 55322.9618 & -47.34 & 55972.6245 & -42.75 \\
\hline \multicolumn{6}{|c|}{ CORAVEL } \\
\hline 54747.3690 & -39.40 & 55095.4120 & -38.90 & 55532.3040 & -41.20 \\
\hline 54748.3000 & -40.40 & 55120.3360 & -44.60 & 55712.5140 & -35.80 \\
\hline 54749.4260 & -38.70 & 55124.3340 & -45.05 & 55719.4970 & -38.10 \\
\hline 54764.4260 & -41.40 & 55138.2500 & -43.30 & 55755.4370 & -42.30 \\
\hline 54814.3500 & -40.00 & 55220.1830 & -39.50 & 55772.4990 & -41.10 \\
\hline 54815.2570 & -40.50 & 55221.1810 & -39.60 & 55777.4870 & -40.70 \\
\hline 54864.1970 & -40.40 & 55346.5130 & -41.00 & 55778.5180 & -40.70 \\
\hline 54866.1960 & -40.40 & 55365.4980 & -39.60 & 55800.5230 & -38.70 \\
\hline 54929.5890 & -45.70 & 55394.4950 & -44.20 & 55801.4590 & -39.00 \\
\hline 54942.5640 & -44.80 & 55395.5130 & -44.70 & 55808.4860 & -38.80 \\
\hline 54947.5480 & -45.10 & 55399.5210 & -43.50 & 55815.4510 & -39.50 \\
\hline 54950.5560 & -44.10 & 55400.5260 & -46.00 & 55816.3910 & -40.00 \\
\hline 55000.4800 & -39.00 & 55444.4920 & -38.60 & 55826.4830 & -41.70 \\
\hline 55013.4660 & -41.50 & 55446.5340 & -39.10 & 55830.3770 & -43.10 \\
\hline 55027.5050 & -39.50 & 55448.5400 & -38.30 & 55850.4120 & -42.35 \\
\hline 55030.4750 & -39.60 & 55463.4160 & -40.50 & 55852.4220 & -41.80 \\
\hline 55037.5250 & -38.00 & 55470.4480 & -43.40 & 55853.3290 & -42.30 \\
\hline 55069.4300 & -40.20 & 55476.4080 & -43.70 & 55860.3200 & -39.60 \\
\hline 55076.4290 & -39.30 & 55477.3080 & -46.50 & 55861.3980 & -41.30 \\
\hline 55077.3640 & -38.50 & 55482.4150 & -44.70 & 55875.3610 & -37.60 \\
\hline 55092.4850 & -37.60 & 55485.3580 & -44.60 & 55881.3830 & -37.60 \\
\hline 55093.3480 & -37.50 & 55496.2960 & -42.40 & 55969.2210 & -39.80 \\
\hline \multicolumn{6}{|c|}{ Hermes } \\
\hline 55023.6954 & -39.91 & 55767.6820 & -41.17 & 55872.4665 & -37.58 \\
\hline 55043.6739 & -41.23 & 55791.6949 & -39.06 & 55873.4721 & -37.46 \\
\hline 55045.6578 & -41.63 & 55828.5818 & -42.37 & 55881.3580 & -38.21 \\
\hline 55082.5616 & -37.26 & 55841.4558 & -43.04 & 55882.3858 & -38.59 \\
\hline 55106.5933 & -42.93 & 55842.4490 & -43.12 & 55883.4060 & -38.81 \\
\hline 55168.4603 & -40.99 & 55852.4188 & -42.09 & 55884.4160 & -39.02 \\
\hline 55394.7242 & -45.04 & 55862.4199 & -39.66 & 55886.3551 & -39.13 \\
\hline 55413.6809 & -43.12 & 55866.4695 & -38.99 & 55888.4476 & -38.99 \\
\hline 55429.5996 & -41.78 & 55867.4144 & -38.64 & 55889.4222 & -38.97 \\
\hline 55474.6315 & -45.73 & 55868.4160 & -38.57 & 55934.3511 & -44.37 \\
\hline 55502.4670 & -41.36 & 55870.4351 & -37.93 & 55937.3255 & -44.09 \\
\hline 55760.6214 & -42.20 & 55871.4217 & -37.70 & 55959.3281 & -41.13 \\
\hline
\end{tabular}


Table 6

Radial Velocity Observations of IRAS 22272+5435

\begin{tabular}{|c|c|c|c|c|c|}
\hline HJD-2,400,000 & $\begin{array}{c}V_{r} \\
\left(\mathrm{~km} \mathrm{~s}^{-1}\right)\end{array}$ & HJD-2,400,000 & $\begin{array}{c}V_{r} \\
\left(\mathrm{~km} \mathrm{~s}^{-1}\right)\end{array}$ & HJD-2,400,000 & $\begin{array}{c}V_{r} \\
\left(\mathrm{~km} \mathrm{~s}^{-1}\right)\end{array}$ \\
\hline \multicolumn{6}{|c|}{ DAO-RVS } \\
\hline $47445.8630^{\mathrm{a}}$ & -39.65 & 48627.6698 & -39.49 & 49177.9472 & -33.91 \\
\hline $47763.9090^{\mathrm{a}}$ & -37.42 & 48719.9755 & -40.06 & 49189.8065 & -36.55 \\
\hline $47789.7860^{a}$ & -35.13 & 48733.9496 & -39.76 & 49217.9122 & -37.57 \\
\hline $48089.8730^{\mathrm{a}}$ & -41.98 & 48756.9420 & -37.56 & 49239.9008 & -39.47 \\
\hline $48467.9460^{\mathrm{a}}$ & -40.55 & 48779.9443 & -38.93 & 49263.9136 & -40.48 \\
\hline 48469.9154 & -39.46 & 48788.9657 & -37.96 & 49286.9238 & -35.72 \\
\hline 48470.9584 & -38.51 & 48799.9447 & -39.32 & 49554.9153 & -38.48 \\
\hline 48471.9298 & -39.04 & 48837.8984 & -38.67 & 49555.9754 & -38.49 \\
\hline 48510.8982 & -39.09 & 48875.8749 & -38.78 & 49899.9641 & -35.84 \\
\hline 48532.8379 & -36.89 & 48886.7604 & -36.41 & 49913.9708 & -36.21 \\
\hline 48533.8327 & -36.50 & 48922.8231 & -33.79 & 49940.8052 & -36.12 \\
\hline 48566.7707 & -33.79 & 49130.9400 & -36.10 & 50020.8213 & -38.46 \\
\hline 48588.7761 & -37.08 & 49163.9505 & -33.76 & $\ldots$ & $\ldots$ \\
\hline \multicolumn{6}{|c|}{ DAO-CCD } \\
\hline 54327.9436 & -38.22 & 54796.6802 & -39.16 & 55406.9180 & -39.54 \\
\hline 54338.8345 & -40.66 & 54945.9563 & -41.91 & 55426.9012 & -38.16 \\
\hline 54354.9248 & -42.43 & 55014.8608 & -43.75 & 55432.8985 & -37.34 \\
\hline 54384.7966 & -42.12 & 55028.8458 & -43.48 & 55476.7658 & -42.91 \\
\hline 54391.7832 & -44.26 & 55041.8470 & -42.30 & 55482.8333 & -41.69 \\
\hline 54434.7340 & -38.41 & 55050.9506 & -42.12 & 55524.6949 & -42.22 \\
\hline 54658.9572 & -41.89 & 55055.9536 & -42.81 & 55719.8822 & -40.89 \\
\hline 54690.9112 & -42.31 & 55063.9118 & -42.93 & 55812.8778 & -42.59 \\
\hline 54709.9258 & -40.27 & 55076.9132 & -41.76 & 55880.7320 & -40.87 \\
\hline 54719.9920 & -39.04 & 55096.8600 & -39.90 & 55881.6898 & -40.92 \\
\hline 54725.9834 & -38.84 & 55167.7288 & -41.66 & 55897.7456 & -38.59 \\
\hline 54741.0134 & -38.95 & 55309.9507 & -37.01 & 55908.7460 & -38.50 \\
\hline 54749.8461 & -38.98 & 55322.9346 & -37.36 & 55938.6723 & -40.21 \\
\hline 54754.8387 & -38.49 & 55385.8899 & -38.99 & 55972.6684 & -40.16 \\
\hline 54790.7215 & -39.00 & 55392.8860 & -40.14 & $\ldots$ & $\ldots$ \\
\hline \multicolumn{6}{|c|}{ CORAVEL } \\
\hline 54747.3470 & -36.50 & 55220.1920 & -37.90 & 55712.5220 & -38.00 \\
\hline 54748.2940 & -36.90 & 55221.1890 & -37.90 & 55719.5100 & -39.80 \\
\hline 54749.4310 & -37.40 & 55302.5870 & -34.60 & 55755.4500 & -38.10 \\
\hline 54764.4330 & -39.20 & 55346.5250 & -41.50 & 55772.5170 & -37.50 \\
\hline 54814.3590 & -38.50 & 55365.5080 & -40.00 & 55777.4960 & -39.20 \\
\hline 54815.2630 & -37.90 & 55394.5070 & -40.30 & 55778.5300 & -39.30 \\
\hline 54864.2080 & -37.80 & 55395.5210 & -39.80 & 55800.5130 & -42.10 \\
\hline 54866.2050 & -38.20 & 55399.5290 & -38.00 & 55801.4490 & -43.00 \\
\hline 54929.5980 & -41.80 & 55400.5330 & -39.20 & 55808.4930 & -39.80 \\
\hline 54940.5850 & -41.30 & 55435.4770 & -34.70 & 55815.4610 & -39.30 \\
\hline 54942.5720 & -40.90 & 55444.4970 & -35.40 & 55816.4010 & -39.00 \\
\hline 54947.5580 & -39.60 & 55446.5430 & -36.70 & 55826.4060 & -38.40 \\
\hline 54950.5640 & -40.40 & 55448.5490 & -36.60 & 55830.3660 & -40.90 \\
\hline 55000.4940 & -44.40 & 55463.4270 & -39.50 & 55852.4330 & -42.00 \\
\hline 55013.4750 & -42.10 & 55470.4590 & -41.40 & 55853.3390 & -43.90 \\
\hline 55027.5130 & -41.20 & 55473.5210 & -40.10 & 55860.3300 & -43.10 \\
\hline 55030.4820 & -41.80 & 55476.4210 & -41.50 & 55861.4080 & -43.10 \\
\hline 55037.5350 & -39.30 & 55477.3320 & -41.20 & 55875.3750 & -41.40 \\
\hline 55069.4380 & -41.10 & 55482.4210 & -40.30 & 55881.3930 & -39.40 \\
\hline 55076.4360 & -40.30 & 55485.3650 & -40.90 & 55969.2070 & -38.70 \\
\hline 55077.3690 & -40.70 & 55496.3150 & -39.40 & 55974.2210 & -38.20 \\
\hline 55092.4930 & -37.90 & 55532.3190 & -40.60 & 55994.2390 & -41.00 \\
\hline 55093.4240 & -36.90 & 55671.5830 & -40.00 & 55995.2310 & -40.60 \\
\hline 55095.4180 & -37.30 & 55675.5740 & -38.60 & 56029.5940 & -36.00 \\
\hline 55120.3500 & -38.00 & 55693.5480 & -37.20 & 56035.5860 & -35.00 \\
\hline 55124.3210 & -38.60 & 55696.5380 & -36.70 & $\ldots$ & $\ldots$ \\
\hline 55138.2570 & -40.90 & 55707.5270 & -36.80 & $\ldots$ & $\ldots$ \\
\hline \multicolumn{6}{|c|}{ Hermes } \\
\hline 55003.6518 & -43.10 & 55760.6111 & -38.43 & 55873.4816 & -42.55 \\
\hline 55031.6428 & -41.20 & 55767.6247 & -38.18 & 55881.5364 & -41.09 \\
\hline 55051.6155 & -42.93 & 55791.7112 & -44.83 & 55882.3729 & -40.83 \\
\hline
\end{tabular}


Table 6

(Continued)

\begin{tabular}{lccccc}
\hline \hline HJD-2,400,000 & $\begin{array}{c}V_{r} \\
\left(\mathrm{~km} \mathrm{~s}^{-1}\right)\end{array}$ & HJD-2,400,000 & $\begin{array}{c}V_{r} \\
\left(\mathrm{~km} \mathrm{~s}^{-1}\right)\end{array}$ & HJD-2,400,000 & $\begin{array}{c}V_{r} \\
\left(\mathrm{~km} \mathrm{~s}^{-1}\right)\end{array}$ \\
\hline 55083.5700 & -39.50 & 55828.5719 & -38.90 & 55883.3813 & -40.83 \\
55106.6049 & -35.68 & 55841.4668 & -41.16 & 55884.4060 & -40.62 \\
55125.4671 & -40.33 & 55842.4580 & -41.37 & 55886.3125 & -40.43 \\
55168.4443 & -40.47 & 55851.4109 & -44.69 & 55887.4003 & -40.28 \\
55217.3265 & -38.49 & 55862.4104 & -44.73 & 55888.4352 & -40.05 \\
55347.7349 & -39.69 & 55866.4789 & -44.08 & 55889.3846 & -39.87 \\
55395.6933 & -40.21 & 55867.4048 & -43.74 & 55903.3333 & -38.31 \\
55421.6207 & -36.97 & 55868.4066 & -43.64 & 55932.3418 & -37.97 \\
55470.5451 & -42.58 & 55870.4257 & -43.18 & 55937.3359 & -38.45 \\
55474.6014 & -42.45 & 55871.4120 & -42.95 & 55959.3548 & -39.28 \\
55502.4764 & -40.27 & 55872.4518 & -42.66 & $\cdots$ & $\cdots$ \\
\hline
\end{tabular}

Note. ${ }^{\text {a }}$ Observations made by R. D. McClure using a K-star mask.

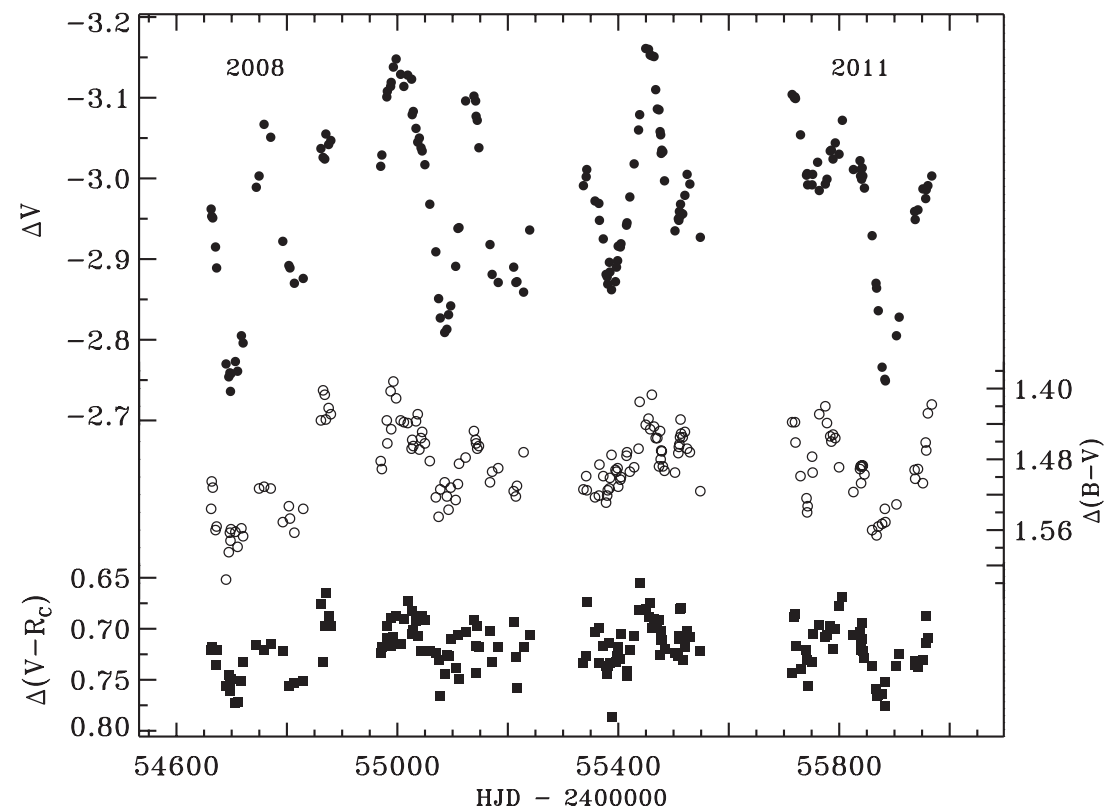

Figure 1. Differential $V$ light curve and $(B-V)$ and $\left(V-R_{C}\right)$ color curves of IRAS $22272+5435$ from the 2008 through 2011 seasons.

$0.49 \mathrm{mag}(1998,2007)$ to $0.22 \mathrm{mag}$ (1995). The changing amplitude of the cyclical variation resembles a beat period and suggests a second period of similar value. The object is redder when fainter. This data set consists of $248 \mathrm{~V}, 189 R_{C}$, and 132 $\left(V-R_{C}\right)$ data points. There is a gap in the $V$ data from 2000 through 2002. The light and color curves are displayed in that paper and the availability of the data is described there.

Our new $B V R_{C}$ light curves from 2008 to 2011 show similar cyclical variations with an average $V$ amplitude of $\sim 0.34$ mag, a larger amplitude in $B(\sim 0.41 \mathrm{mag})$, and a slightly smaller amplitude in $R_{C}(\sim 0.31)$. These new light and color curves are shown in Figure 1. The depths of the minima vary quite a bit, from being similar in 2009 to differing by a factor of about three in 2011. The pattern differs from the regular pattern of deep minimum followed by shallow minimum seen in RV Tauri variables. The color curves mimic the cyclical variations in the light curve, with the object again seen to be redder when fainter. This can also be seen clearly in Figure 2, a plot of brightness versus color. These data show good continuity with our older data; the comparison star data appear to be consistent and the light and color curves show similar average values and ranges of variability.

Arkhipova et al. $(1993,2000)$ have published photometric studies of IRAS $22272+5435$ from 1991 to 1999 and made their $U B V$ data available publicly, which consist of 89 observations in each filter. There is thus overlap between the two data sets from 1994 to 1999.

To investigate further the variability in the system and to search for multiple periods or changing periods, we have combined our two sets of $V$ observations together with the $V$ data of Arkhipova et al. (1993, 2000). In Paper I we published differential $V$ magnitudes with respect to a comparison star, as we have done here, although using a different comparison star. Using the standard magnitudes of the comparison stars $\left(V\left(\mathrm{C}_{1, \text { new }}\right)=\right.$ 11.62; $\left.V\left(\mathrm{C}_{1, \text { old }}\right)=11.16\right)$, we have formed $V$ magnitudes for our observations of IRAS $22272+5435$. Arkhipova et al. published their light curve data in the form of $V$ magnitudes.

An initial combination of the data revealed a systematic offset when plotted, with the data of Arkhipova et al. (1993, 2000) brighter than ours. We then compared the data on nights in 

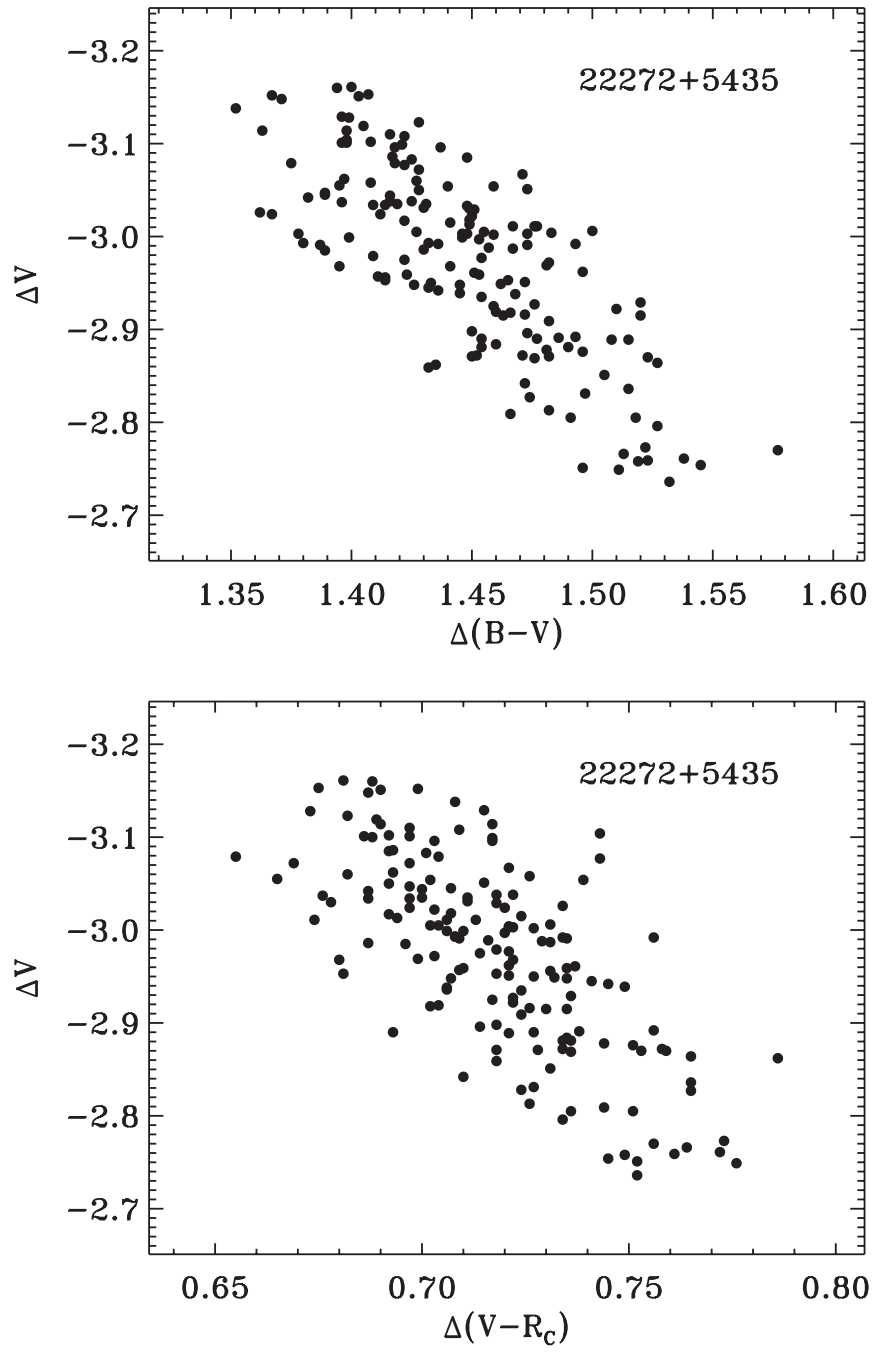

Figure 2. Brightness vs. color curves for the new observations of IRAS $22272+5435$. They clearly show the correlation of color with brightness; the object is redder when fainter.

which we both observed the object (nine nights in 1995-1998), and found an offset of $0.105 \pm 0.006 \mathrm{mag}$. Several factors could contribute to such an offset, such as a variation in the comparison stars or inclusion of another star in the aperture. ${ }^{8}$ For the purposes of this period study, we have added an offset of $+0.105 \mathrm{mag}$ to each of the $V$ magnitudes of Arkhipova et al. $(1993,2000)$ to bring the two data sets into agreement. The resulting combined $V$ light curve is shown in Figure 3. The combined $V$ light curve displays the variations in seasonal

\footnotetext{
8 We investigated several factors which could contribute to an offset in the two data sets, beginning with a possible variation in the comparison star. We have four standardized observations of our initial comparison star made over a 17 year interval, and they agree to within $\pm 0.015 \mathrm{mag}$ and our comparison stars appear to be constant at the level of \pm 0.015 mag over the 18 seasons of our observations. This leads us to think that there is not a systematic zero-point error in our data. The comparison star used by Arkhipova et al., BD+54 2793 (HD 235865), has $B-V=1.91$ and is classified as M2 III (SIMBAD), so it is easy to suspect that it varies at some level. Arkhipova et al. (1993) list a value of $V=8.54$ while in SIMBAD it is listed at $V=8.60$. So the cause could be a variation in their comparison star. They used a photoelectric photometer with a large aperture of $27^{\prime \prime}$ diameter, which thus includes a star near IRAS $22272+5435$; we measured that the effect of this would be to increase the brightness attributed to IRAS $22272+5435$ of $\sim 0.015$ mag. Some combination of these factors and the uncertainty of \pm 0.015 mag in our standard star measurements likely explains the systematic difference in brightness of the two data sets.
}

amplitude and in seasonal mean brightness seen previously in our data alone. The variations in seasonal amplitude are due to the presence of multiple pulsation periods, as will be discussed shortly, which can enhance or diminish the amplitude. The variations in mean seasonal brightness might be due to temporal changes in the circumstellar opacity, since the star is known to be surrounded by a dusty nebula.

The combined $V$ data set and the VUO $R_{C}$ and $\left(V-R_{C}\right)$ data sets were searched for periodicity using the program Period04 (Lenz \& Breger 2005). We explored multiple periods and found at least four significant periods in most of the data sets. A period was judged to be significant if the $\mathrm{S} / \mathrm{N}$ amplitude ratio of the peak in the frequency power spectrum was $\geqslant 4$ (Breger et al. 1993). In light of the aforementioned seasonal variations in the mean light level, we began by comparing the results of a period analysis based on the observed data with one in which the observations of each season were normalized to the mean brightness of the entire data set. This was carried out for the combined $V$ light curve. The results were that the dominant first period and also the second period were the same in both cases. The next two differed, but there was no evidence of a periodicity in the seasonal means. Thus we will report the period study based on the seasonally normalized $V, R_{C}$, and $V-R_{C}$ data, with the assumption that this better represents the pulsational properties of the object. The results are as follows and are tabulated in Table 7 .

A. The combined V data set from 1991 to 2011. For the combined $V$ data set, there is a dominant period of $131.9 \pm$ 0.1 days with a second period of $125.0 \pm 0.1$ days. The combination of these two periods gives a reasonably good fit to the modulated light curve. The ratio of these periods is $P_{2} / P_{1}=0.95$. The power spectrum of the first period is shown in Figure 4, along with a phase diagram from this single period. Comparing the Fourier components of the fit with four periods to the light curve results in somewhat better agreement than does the fit with two; this fit is shown in the bottom panel of Figure 3. $P_{4}$ is somewhat uncertain and an almost equally good value is 155.9 days, which equals $2 / 3$ of $P_{4}$.

B. The combined $V$ data set from 1991 to 1999. The combined $V$ data from the first nine years yield a similar value for $P_{1}$ but do not contain the period of 125 days seen as $P_{2}$ in the entire data set.

C. The V data set of the VUO from 2002 to 2011. The $V$ data from the last 10 years yield values for $P_{1}$ and $P_{2}$ that are similar to those found for the entire data set (set A above). The values for $P_{3}$ and $P_{4}$ are $\sim 5 \%$ and $\sim 10 \%$ larger than those for the entire data set, respectively, with $P_{4} \approx 2 P_{2}$.

D. The $R_{C}$ data set of the VUO from 2002 to 2011. The $R_{C}$ data from the last 10 years were also investigated. They yield values of $P_{1}$ and $P_{2}$ similar to those found for the corresponding $V$ data set and for the entire $V$ data set. However, the values of $P_{3}$ and $P_{4}$ are different, and a value for $P_{4}$ of 222.6 days is almost as significant as that of 138.1 days as listed. The amplitude of the $R_{C}$ light curve is significantly smaller than the $V$ light curve, and this makes especially the weaker periods less certain.

E. The $R_{C}$ data set of the VUO from 1999 to 2011 . The $R_{C}$ data beginning in 1999, the first year in which we obtained a reasonable number of data points, were also examined. This 13 year data set yielded values for the periods similar to those found for the slightly more restricted $R_{C}$ data set 


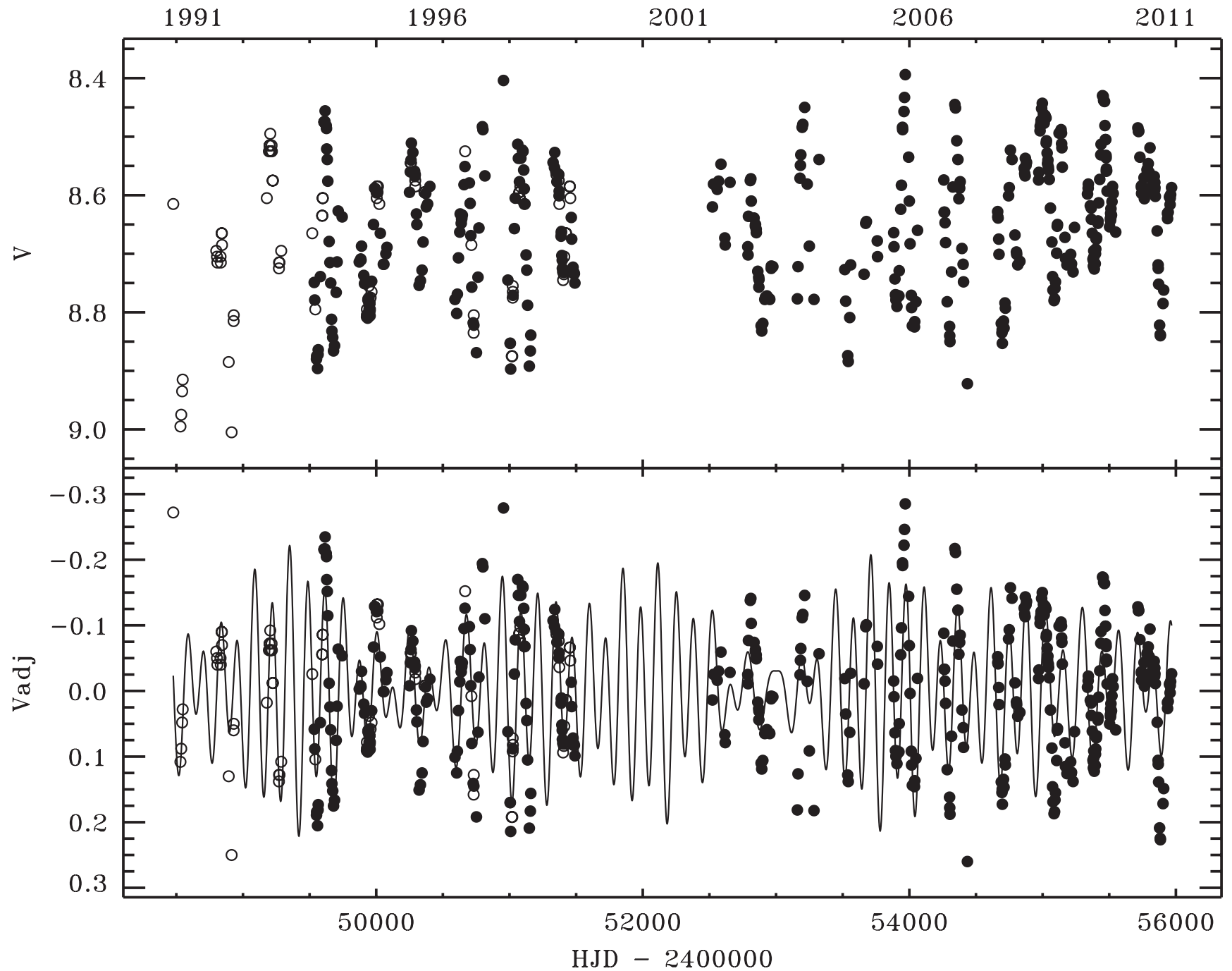

Figure 3. Top: the combined $V$ light curve of IRAS $22272+5435$ from the 1991 to 2011 seasons. Bottom: the seasonally adjusted, combined $V$ light curve fitted with the four periods and amplitudes of the periodogram analysis. The filled circles are the data from the VUO and the open circles are from Arkhipova et al. (1993, 2000).

Table 7

Periodogram Study of the Light and Color Curves of IRAS 22272+5435

\begin{tabular}{|c|c|c|c|c|c|c|c|c|c|c|c|c|c|c|c|}
\hline $\begin{array}{l}\text { Data } \\
\text { Set }\end{array}$ & Filter & Years & $\begin{array}{l}\text { No. } \\
\text { Obs. }\end{array}$ & $\begin{array}{c}P_{1} \\
\text { (days) }\end{array}$ & $\begin{array}{c}A_{1} \\
(\mathrm{mag})\end{array}$ & $\phi_{1}{ }^{b}$ & $\begin{array}{c}P_{2} \\
\text { (days) }\end{array}$ & $\begin{array}{c}A_{2} \\
(\mathrm{mag})\end{array}$ & $\phi_{2}{ }^{b}$ & $\begin{array}{c}P_{3} \\
\text { (days) }\end{array}$ & $\begin{array}{c}A_{3} \\
(\mathrm{mag})\end{array}$ & $\phi_{3}{ }^{b}$ & $\begin{array}{c}P_{4} \\
\text { (days) }\end{array}$ & $\begin{array}{c}A_{4} \\
(\mathrm{mag})\end{array}$ & $\phi_{4}{ }^{\mathrm{b}}$ \\
\hline A & V & $1991-2011$ & 507 & 131.9 & 0.097 & 0.48 & 125.0 & 0.048 & 0.94 & 145.4 & 0.044 & 0.48 & 229.5 & 0.042 & 0.97 \\
\hline B & V & 1991-1999 & 230 & 131.4 & 0.110 & 0.42 & 146.7 & 0.055 & 0.66 & 225.0 & 0.042 & 0.84 & 82.9 & 0.035 & 0.20 \\
\hline D & $R_{C}$ & $2002-2011$ & 287 & 131.2 & 0.076 & 0.16 & 125.8 & 0.058 & 0.23 & 164.4 & 0.045 & 0.32 & 138.1 & 0.039 & 0.78 \\
\hline $\mathrm{E}$ & $R_{C}$ & 1999-2011 & 335 & 130.7 & 0.064 & 0.97 & 126.0 & 0.048 & 0.32 & 164.7 & 0.040 & 0.41 & 138.7 & 0.037 & 0.01 \\
\hline $\mathrm{F}$ & $V-R_{C}$ & $2002-2011$ & 265 & 130.3 & 0.023 & 0.88 & 124.2 & 0.013 & 0.65 & 138.1 & 0.012 & 0.69 & $\ldots$ & $\ldots$ & $\ldots$ \\
\hline
\end{tabular}

Notes.

${ }^{a}$ The uncertainties in $P, A, \phi$ are approximately \pm 0.3 days, $\pm 0.005 \mathrm{mag}, \pm 0.015$, respectively, except for data set A, in which the uncertainty in $P$ is \pm 0.1 days, and set $\mathrm{F}$, in which the uncertainty in $A$ is \pm 0.002 .

$\mathrm{b}$ The phases are determined based on the epoch of 2,448,000.00.

from 2002 to 2011, with $P_{1}$ and $P_{2}$ close to those found for the combined $V$ data set.

F. The $V-R_{C}$ data set of the VUO from 2002 to 2011. The $V-R_{C}$ data from the last 10 years yield values for $P_{1}$ and $P_{2}$ that are close ( $<1 \%$ difference) to those found from the other data sets. Thus they show clearly that a similar periodicity is found in the color curve as in the light curves.
These results show a consistent primary period of 131 days throughout this 20 year observing interval, with no indication of a secular change in the primary period. The value of $P_{2}=$ 125 days, however, is not found in the 1991-1999 data set, although it is seen in all of the data sets from 1999 to 2011 and in the combined 1991-2011 V data set. These values yield a ratio of $P_{2} / P_{1}=0.95$. 

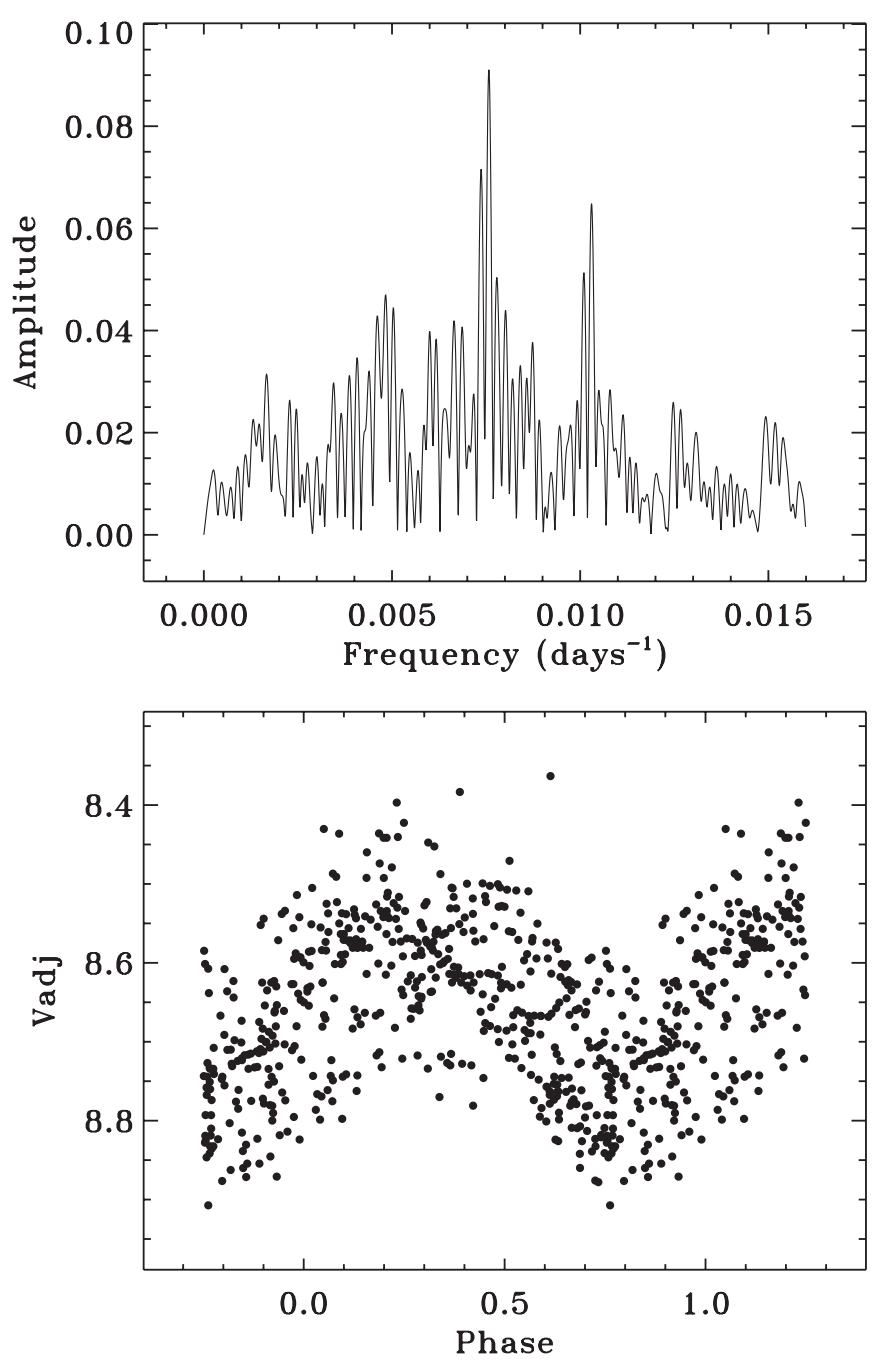

Figure 4. Top: the frequency spectrum for the first period of the $V$ light curve of IRAS $22272+5435$. Bottom: the phase plot of the seasonally normalized data based on the frequency peak, $P_{1}=131.9$ days.

To investigate further any evidence for a secular change in the period, we plot in Figure 5 the cycle number versus Heliocentric Julian Date (HJD) for the minima of IRAS 22272+5435. This is based on visual inspection of the VUO $V$ and $R_{C}$ light curves. On this scale, the data points appear to fit a straight line, with a slope yielding a period of 131.9 days, the main period determined from the periodogram analysis. Also plotted are the residuals from this straight-line fit. They show the minima initially occurring later than predicted, but then gradually occurring earlier, as if the period were shortening, then later as if the period were lengthening, then occurring earlier. This suggests a cyclical pattern with a period of $\sim 34$ cycles or $\sim 4500$ days (12.3 years), although future data are needed to confirm that the minima are occurring later than predicted by the linear ephemeris. An obvious thought is that this is showing the longer-term beat period. However, based on $P_{1}=131.9$ days and $P_{2}=125.0$ days, the beat period is 2389 days, while the apparent period seen in the residuals is almost twice that value. We compared these residuals with those derived from a linear fit to the times of minimum of the theoretical $V$ light curve based on the four periods and their amplitudes, as shown in Figure 3. This resulted in residuals of similar $(O-C)$ range but which did not show the same pattern.

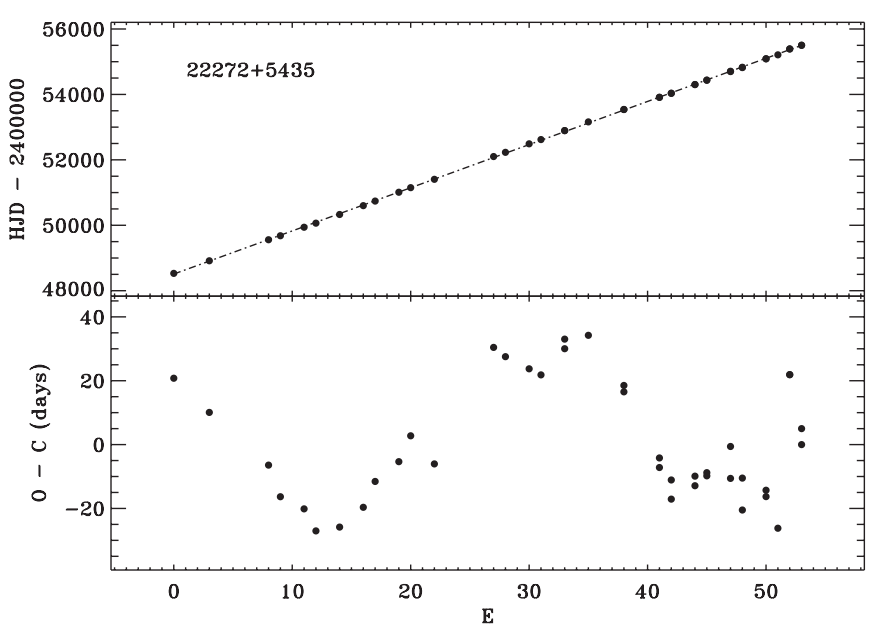

Figure 5. Top: observed time of minimum vs. cycle count for the $V$ and $R_{C}$ light curves of IRAS $22272+5435$. The slope of the line is 131.9 days cycle ${ }^{-1}$. Bottom: the residuals of the time of minimum about the straight-line fit.

\subsection{Radial Velocity Study}

As described earlier, we have carried out a long-term series of radial velocity observations of IRAS $22272+5435$, initially from 1991 to 1995 , and then resuming in 2005 and continuing to the present time. Recently, two of us published an intensive radial velocity study with observations from 2005 to 2007, especially from the 2006 season (Začs et al. 2009).

Initial examination of the more recent CORAVEL and DAO-CCD observations for IRAS $22272+5435$ showed an apparent offset in the velocities observed in the same seasons. Thus we decided to explore this further by comparing the radial velocity measurements of IRAS $22272+5435$ from the three observatories. We compared the differences (1) in the average values of the velocities from each observatory for all nights, (2) in the average values of the velocities only over the years in which they were observed in common at the different observatories, (3) in the individual velocities observed on common nights or successive nights at two different observatories, and (4) in the systemic velocities $\left(V_{0}\right)$ when we were able to compute velocity solutions for the data from an individual observatory. Each of these methods of comparison has its limitations: (1) the yearly averages are not necessarily the same from year to year, (2) and even within a year, the different distribution of observation dates may skew the averages from the different observatories. Method (3) is the best in principle, but the number of nights in one case is small (6). We do not find radial velocity solutions for all of the data sets alone (not for DAO-CCD), so method (4) cannot be applied to each. Comparing the different methods, we arrived at the following offsets, which were added to the different data sets to bring them to the same velocity system for IRAS $22272+5435$, which we arbitrarily set to that of the DAO-CCD observations. The offsets are as follows: $-1.4 \mathrm{~km} \mathrm{~s}^{-1}$ for the CORAVEL measurements and $-0.2 \mathrm{~km} \mathrm{~s}^{-1}$ for the Hermes measurements. The latter may or may not be real, but there is a real, significant difference between the CORAVEL measurements and the other two data sets. ${ }^{9}$

\footnotetext{
9 IRAS $22272+5435$ has an usual spectrum, with strong lines due to $s$-process elements and very strong reddening of its continuum. The three different instruments used for the 2005-2011 measurements each sample different regions of the spectrum and at different resolutions. Thus, it is perhaps not surprising that there are zero-point differences in the measurements for IRAS $22272+5435$, even though each of the different
} 

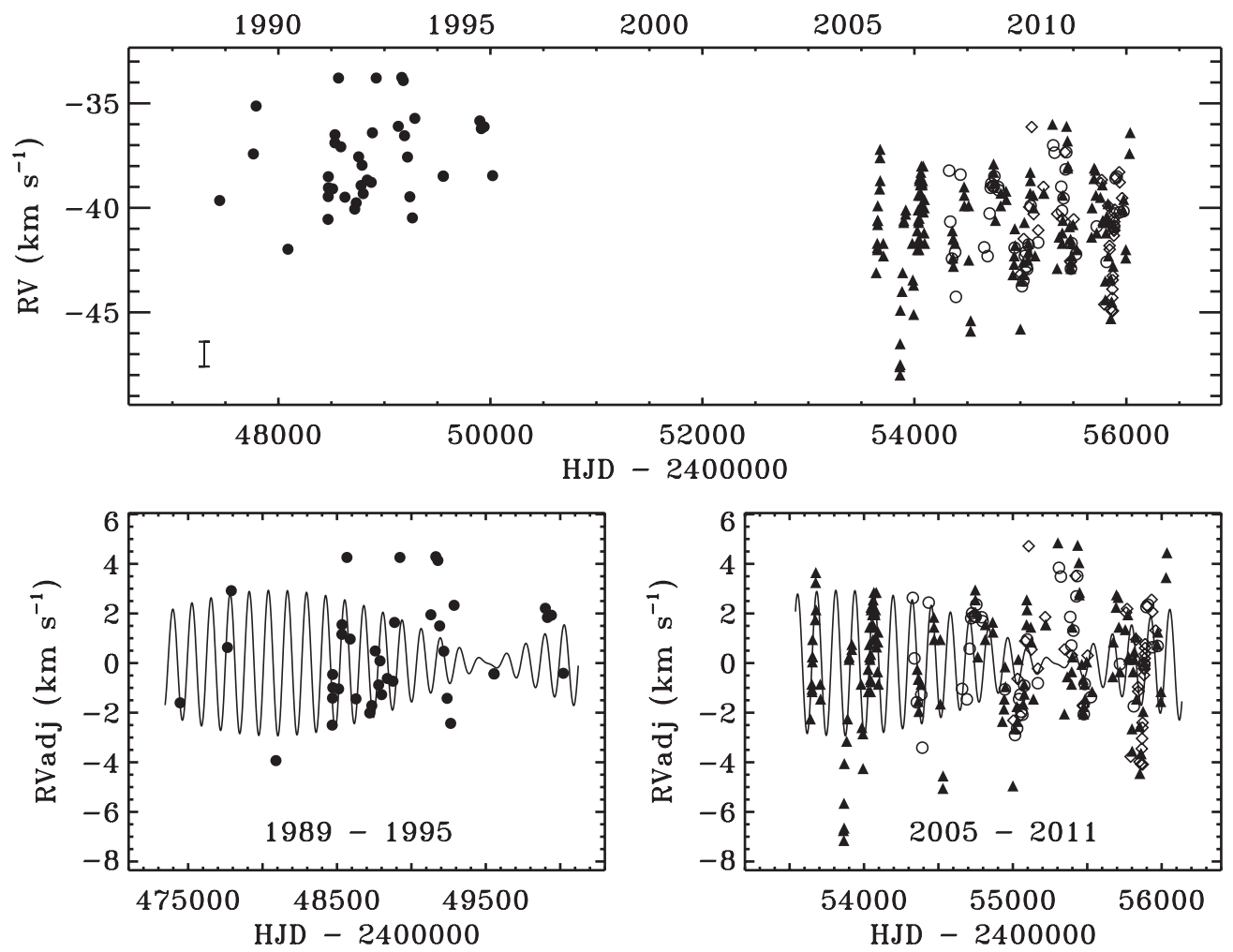

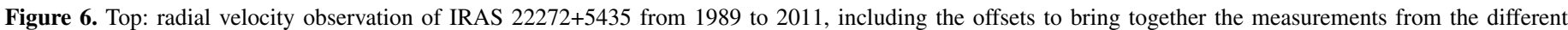

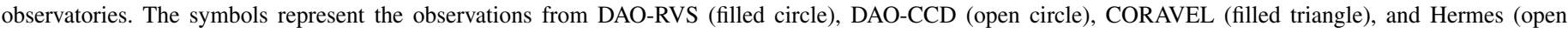

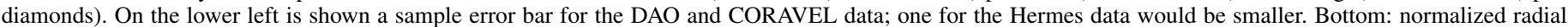

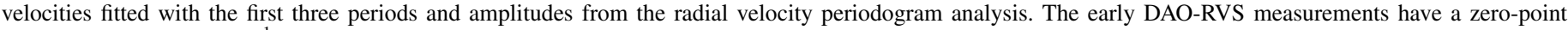
adjustment of $-2.80 \mathrm{~km} \mathrm{~s}^{-1}$ to normalize the data. See the text for more details.

We note that Klochkova et al. (2009) have published four radial velocity observations and Reddy et al. (2002) one observation from 2000 of IRAS $22272+5435$. However, in light of the systematic differences that we have found among our three data sets, we have chosen not to include these few additional observations in our study.

We have combined these data sets with the above offsets to investigate the period and radial velocity properties of IRAS $22272+5435$. This combined radial velocity curve is shown in Figure 6. One can see a variation in the radial velocities that reaches up to $10 \mathrm{~km} \mathrm{~s}^{-1}$ within a season (2006). This is the pulsational variation reported in the preliminary study of Hrivnak \& Lu (2000) and the study of Začs et al. (2009). It can be seen that there also exists a systemic difference between the early velocity measurements from 1988 to 1995 and the later measurements. This has been discussed by Hrivnak et al. (2011) and attributed to a binary companion. Since our goal is to study the pulsational properties of the object, we have removed this difference by normalizing the earlier and later data by a zero-point adjustment to the earlier data $\left(-2.8 \mathrm{~km} \mathrm{~s}^{-1}\right)$. There also exist variations in the mean values from season to season, with, in the extreme cases, the measurements in 2006 extending to more negative values and those from 2010 extending to less negative values than the others. While we saw an analogous effect in the light curves that we attributed to varying opacity in the circumstellar envelope, we have no similar explanation for

velocity systems is well calibrated. This could be investigated further using the spectrum of IRAS $22272+5435$ over the various wavelength regions and simulating the different detector resolutions and analysis methods. However, we have chosen not to do so within the scope of this study. this velocity change and have not carried out a seasonal normalization of the velocity curves as we did for the light curves.

A periodogram analysis was carried out of this combined, normalized radial velocity data set. We analyzed the entire data set and also the earlier and later data separately. The results are listed in Table 8 .

A. The combined radial velocity data set from 1988 to 2011. We found three significant periods: $P_{1}=131.2 \pm 0.1$ days, $P_{2}=125.5 \pm 0.1$ days, and a weaker $P_{3}=66.8 \pm 0.1$ days, a little more than half the value of $P_{1}$. The values of $P_{1}$ and $P_{2}$ are very close to those determined from the combined $V$ light curve. However, for the light curve the period of $\sim 132$ days was clearly more dominant while for the velocity curve the two periods are more similar in amplitude. The fit of the velocity curve with the Fourier components based on three periods gives a reasonably good fit to most of the data. This is shown in the bottom panels of Figure 6. However, the fits are not good in the regions where the sine curves interfere destructively, such as the fit to the 2009-2010 data. Also the fit is not good in 2011, where the two minima in the velocity curve are separated by $\sim 67$ days, the value of $P_{3}$. Note, however, this period of $\sim 67$ days is not found only in the 2011 and perhaps 2010 data; it was found as a secondary period in the 2005-2007 data of Začs et al. (2009).

B. The combined radial velocity data set from 2005 to 2011. For the later data set, one finds periods similar to those found from the entire velocity data set, with $P_{1}=132.7 \pm$ 0.5 days the most dominant. However, when we try to vary all three periods simultaneously to achieve the best overall fit, they do not converge; instead we find the two similar 

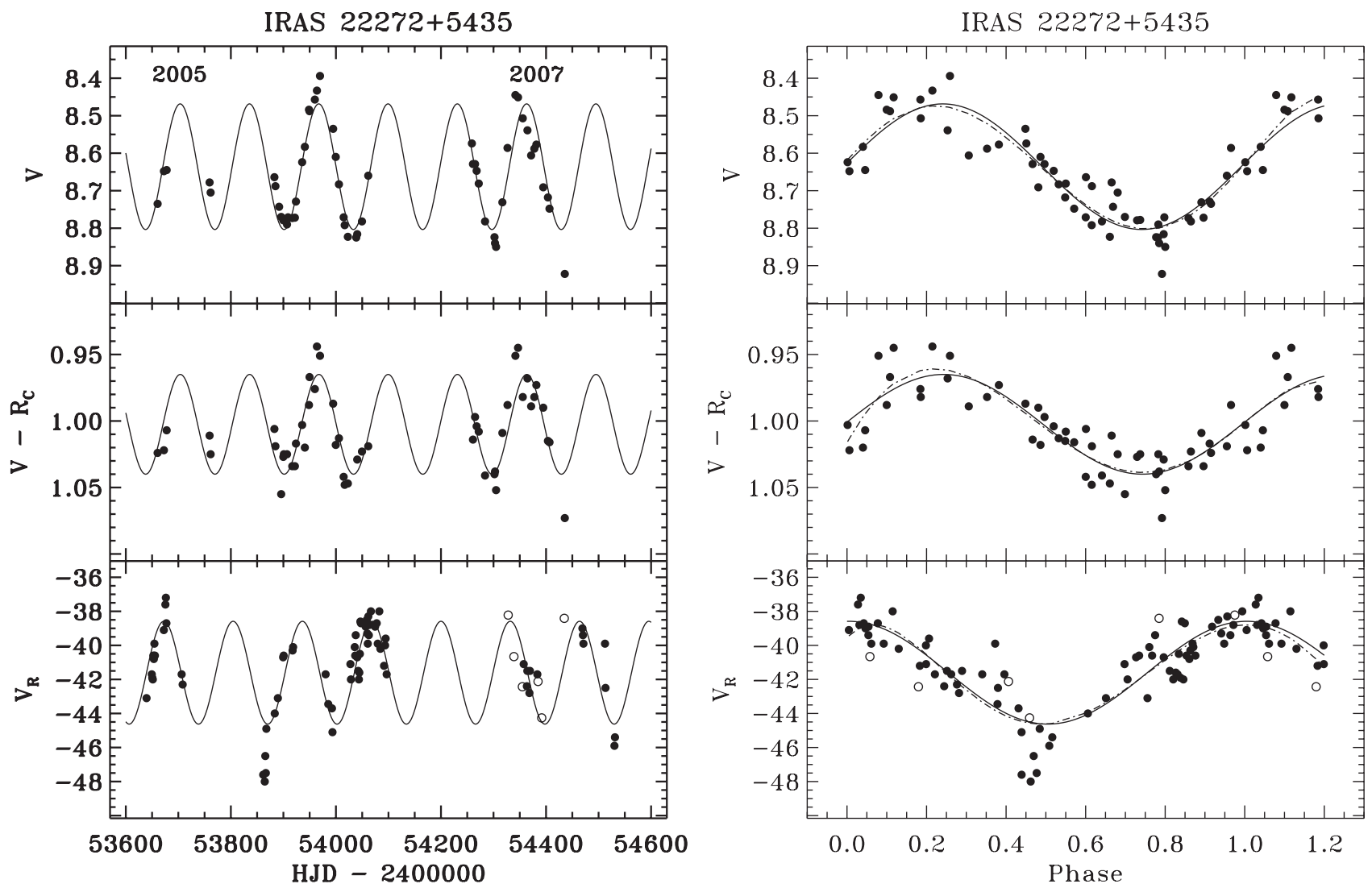

Figure 7. (a, left panel): contemporaneous $V,\left(V-R_{C}\right)$, and $V_{R}$ curves of IRAS $22272+5435$ from 2005 to 2007 , along with sine-curve fits based on $P=131.9$ days. (b, right panel): phased plots ( $P=131.9$ days), along with sine curves (solid lines) and polynomial curves (dashed lines) fitted to the observations. In the radial velocity panels, the filled circles are the data from Začs et al. (2009) and the open circles from the DAO-CCD observations.

Table 8

Periodogram Study of the Radial Velocity Curve of IRAS 22272+5435

\begin{tabular}{|c|c|c|c|c|c|c|c|c|c|c|c|c|}
\hline $\begin{array}{l}\text { Data } \\
\text { Set }\end{array}$ & Years & $\begin{array}{l}\text { No. } \\
\text { Obs. }\end{array}$ & $\begin{array}{c}A_{0} \\
\left(\mathrm{~km} \mathrm{~s}^{-1}\right)\end{array}$ & $\begin{array}{c}P_{1} \\
\text { (days) }\end{array}$ & $\begin{array}{c}A_{1} \\
\left(\mathrm{~km} \mathrm{~s}^{-1}\right)\end{array}$ & $\phi_{1}{ }^{b}$ & $\begin{array}{c}P_{2} \\
\text { (days) }\end{array}$ & $\begin{array}{c}A_{2} \\
\left(\mathrm{~km} \mathrm{~s}^{-1}\right)\end{array}$ & $\phi_{2}{ }^{b}$ & $\begin{array}{c}P_{3} \\
\text { (days) }\end{array}$ & $\begin{array}{c}A_{3} \\
\left(\mathrm{~km} \mathrm{~s}^{-1}\right)\end{array}$ & $\overline{\phi_{3}{ }^{b}}$ \\
\hline A & 1988-2011 & 274 & $-40.77^{\mathrm{c}}$ & 131.2 & 1.52 & 0.96 & 125.5 & 1.42 & 0.93 & 66.8 & 1.01 & 0.98 \\
\hline B & 2005-2011 & 236 & -40.81 & 132.7 & 1.43 & 0.54 & 67.0 & 1.13 & 0.36 & 125.8 & 1.04 & 0.03 \\
\hline $\mathrm{C}$ & 1988-1995 & 38 & $-40.59^{c}$ & 126.2 & 1.83 & 0.95 & 148.5 & 1.43 & 0.35 & $\ldots$ & $\ldots$ & $\ldots$ \\
\hline
\end{tabular}

Notes.

${ }^{a}$ The uncertainties in $P, A, \phi$ for set A are approximately \pm 0.1 days, $\pm 0.15 \mathrm{~km} \mathrm{~s}^{-1}, \pm 0.018$, respectively. They are larger for the other, smaller data sets.

$\mathrm{b}$ The phases are determined based on the epoch of 2,448,000.00.

${ }^{\mathrm{c}}$ The velocities from the 1988-1995 data set were adjusted with an additive offset of $-2.80 \mathrm{~km} \mathrm{~s}^{-1}$ to bring them to approximately the same level as the zero-point adjusted 2005-2011 data.

periods continue to move closer together and the amplitudes continue to increase. The value of $P=67$ days is again found and appears to at least partly derive from the 2011 velocities which have this smaller interval between two velocity minima.

C. The combined radial velocity data set from 1988 to 1995. This is a small data set, with only 39 observations, and the values are less certain, with $P_{1}=126.2 \pm 1.0$ days and $P_{2}=148.5 \pm 1.7$ days. The first of these is a value similar to the value of $P_{2}$ in most of the velocity and light curve analyses. The second is similar to the value of $P_{2}$ found in the analysis of the 1991-1999 $V$ light curve, which covers a somewhat similar set of dates.

We also investigated the fit to the normalized radial velocity curve from 1988 to 2011 using the values of $P_{1}$ and $P_{2}$ fixed at those determined from the combined, normalized $V$ light curve. The fit was good and a third significant period of 66.8 days was found, the same as found in set A above. However, the fit is not quite as good as when the periods are determined from the velocity curve itself.

\subsection{Contemporaneous Light, Color, and Velocity Curve Study}

The availability of these light and velocity observations from the same seasons affords the opportunity to compare contemporaneously the light, color, and velocity curves. We begin with the large number of radial velocity observations obtained by Začs et al. (2009) during the 2006 season, at a time when we also had good light and color curve observations. To enlarge the sample, particularly to include the 2005 radial velocity and the 2007 light and color curve observations, we have combined the data from 2005 to 2007 beginning with the time of the radial velocity observations. These are shown in Figure 7(a). For the subsequent radial velocity analysis, we have used only the Začs et al. (2009) radial velocities and have not 
IRAS $22272+5435$

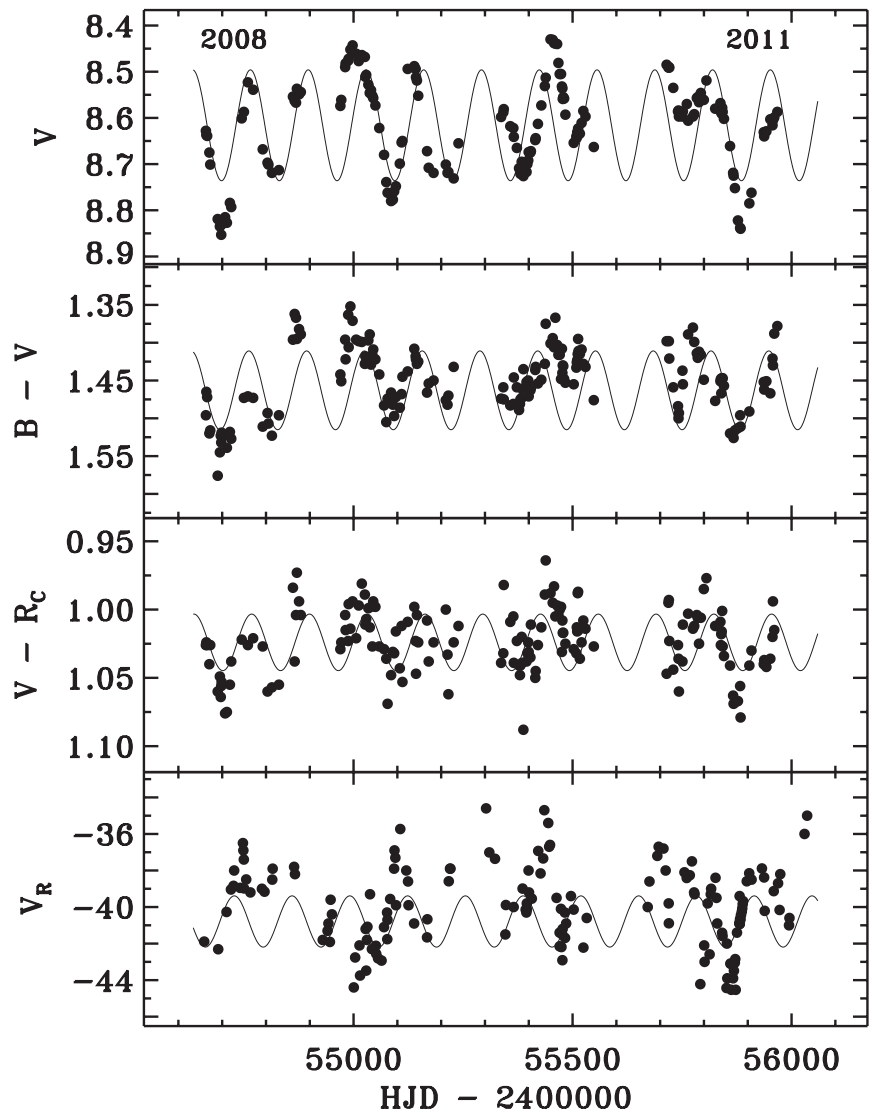

Figure 8. Contemporaneous $V,(B-V),\left(V-R_{C}\right)$, and $V_{\mathrm{R}}$ curves of IRAS $22272+5435$ from 2008 to 2011 , along with sine-curve fits based on $P=131.9$ days and the amplitudes and phases derived from the 2008-2009 data only.

included the six from DAO in 2007, although they are plotted in the figure and show good agreement. Sine curves were fitted to the data using the dominant period determined from the combined $V$ light curve of 131.9 days and an arbitrary epoch of $T_{0}=2,448,000$. This yielded sine-curve values of semiamplitude and phase, respectively, as follows $-V$ : $0.168 \mathrm{mag}$ and $0.51, V-R_{C}: 0.038 \mathrm{mag}$ and 0.51 , and $V_{\mathrm{R}}: 3.02 \mathrm{~km} \mathrm{~s}^{-1}$ and 0.25 , with uncertainties in the phase of \pm 0.01 . Curves fitted with these parameters are overplotted on the observations in Figure 7(a), and they agree well with the observations. One can immediately see that the light and color curves are exactly in phase according to a sine-curve fit, being exactly brightest when bluest. The radial velocity differs by 0.26 in phase, approximately a quarter of a cycle, being at the average velocity and minimum size when the star is brightest.

The data are folded on this period and displayed during one cycle in Figure 7(b). In this figure, in addition to using sine curves, we also fit the phased data with fourth-order polynomial curves. These show that the velocity curve is essentially a sine curve, while the light and color curves appear to peak a bit earlier in phase $(\sim 0.02)$ and have a little more gradual decline to minimum and a little steeper rise to maximum than does a sine curve. Given the dispersion of the observations about the lines, it can be seen that the sine curves represent the observations reasonably well.

We similarly examined the 2008-2011 observations. They are shown in Figure 8 . The light and color curves have been displayed previously in Figure 1 and show considerable variation in the relative depths of minimum light and the mean levels in the different seasons. We have now normalized the seasonal light curves (but not the color curves) to their average values. As mentioned earlier, the 2011 radial velocity observations display a period of $\sim 67$ days, about half of the period found from the rest of the data. This can be seen clearly in this figure. Sine curves were fitted to these observations with $P=131.9$ days and the same epoch as above. We find that the fit to the $V$ light curve is not very good when we include all four years; this is partly due to the large variations in the depths of minimum. Similarly, the color curves are not fit well for all four years; neither is the velocity curve. Examining them over smaller intervals, the best fit is found when restricted to the 2008-2009 interval. For the 2008-2009 observations, we find sine-curve values of semi-amplitude and phase, respectively, as follows $-V$ : $0.120 \mathrm{mag}$ and $0.55, B-V: 0.052 \mathrm{mag}$ and 0.58 , $V-R: 0.021 \mathrm{mag}$ and 0.53 , and $V_{\mathrm{R}}: 1.40 \mathrm{~km} \mathrm{~s}^{-1}$ and 0.33 . The uncertainties in the phases range from 0.02 to 0.03 . The light and color curves appear to again be in phase and the radial velocity curves differ by 0.22 in phase, again about a quarter of a cycle; thus the phasing of the different curves agrees with the results found earlier for the 2005-2007 curves. We have plotted the sine curves with these parameters, derived from the 2008-2009 observations, through all of the 2008-2011 observations shown in Figure 8. It can be seen that the fit is not as good as found for the 2005-2007 observations, when the variations appeared to be less complex. In particular, one can see the poor agreement to the period in the 2011 and perhaps the 2010 radial velocity data. We will examine this in a future study in which we also include additional spectroscopic observations.

\section{VARIABILITY STUDY OF IRAS $22223+4327$}

\subsection{Light Curve Study}

Our earlier $V$ and $R$ light curves of IRAS 22223+4327 from 1994 through 2007 have also been described in Paper I. The light curves show a general decrease in brightness from 1994 to 2007, amounting to $\sim 0.16 \mathrm{mag}$ in $V$. Superimposed on this is a cyclical variation seen in the seasonal light curves. The $V$ amplitude of this cyclical variation changes from $0.21 \mathrm{mag}$ (1997) to $0.09 \mathrm{mag}$ $(2002,2006)$; this is about half of the corresponding range seen in IRAS $22272+5435$. The object is also redder when fainter. A period of $\sim 88$ days is found in the light and color curves. That data set consists of $267 \mathrm{~V}, 212 R_{C}$, and $160\left(V-R_{C}\right)$ data points, with a gap in the $V$ data from 2000 through 2002. The light and color curves are displayed in that paper and the data are made available.

Our new $B V R_{C}$ light curves also show cyclical variations with a large amplitude change that ranges in $V$ from 0.23 mag (2010) to $0.10 \mathrm{mag}$ (2008). The amplitude is larger in $B$, ranging from 0.31 to $0.14 \mathrm{mag}$, and slightly smaller in $R_{C}, 0.19$ to $0.10 \mathrm{mag}$. However, the light curves no longer show a trend of decreasing brightness; rather, the median levels are approximately the same in each year. Our newer and older observations for IRAS 22223+4327 appear to be consistent with each other based on the comparison star data and the color curve data. These new light and color curves are shown in Figure 9. The object is again seen to be redder when fainter, as seen in the color curves in Figure 9 and as shown explicitly in the brightness versus color curves in Figure 10.

Arkhipova et al. $(2003,2011)$ have published photometric studies of IRAS 22223+4327 from 1999 to 2002, consisting of 56 observations in each of the $U B V$ filters, and from 2003 to 


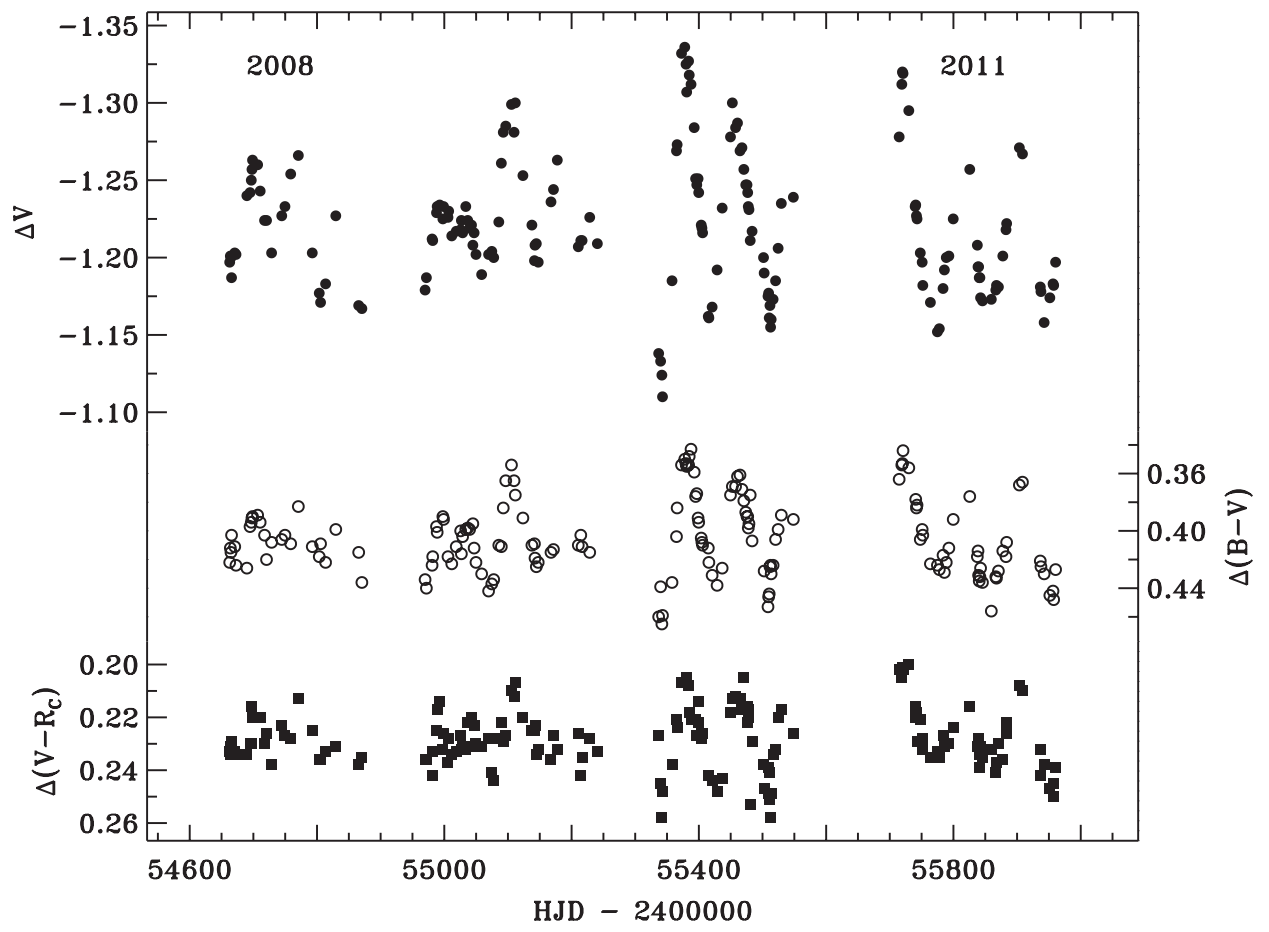

Figure 9. Differential $V$ light curve and $(B-V)$ and $\left(V-R_{C}\right)$ color curves of IRAS 22223+4327 from 2008 to 2011.
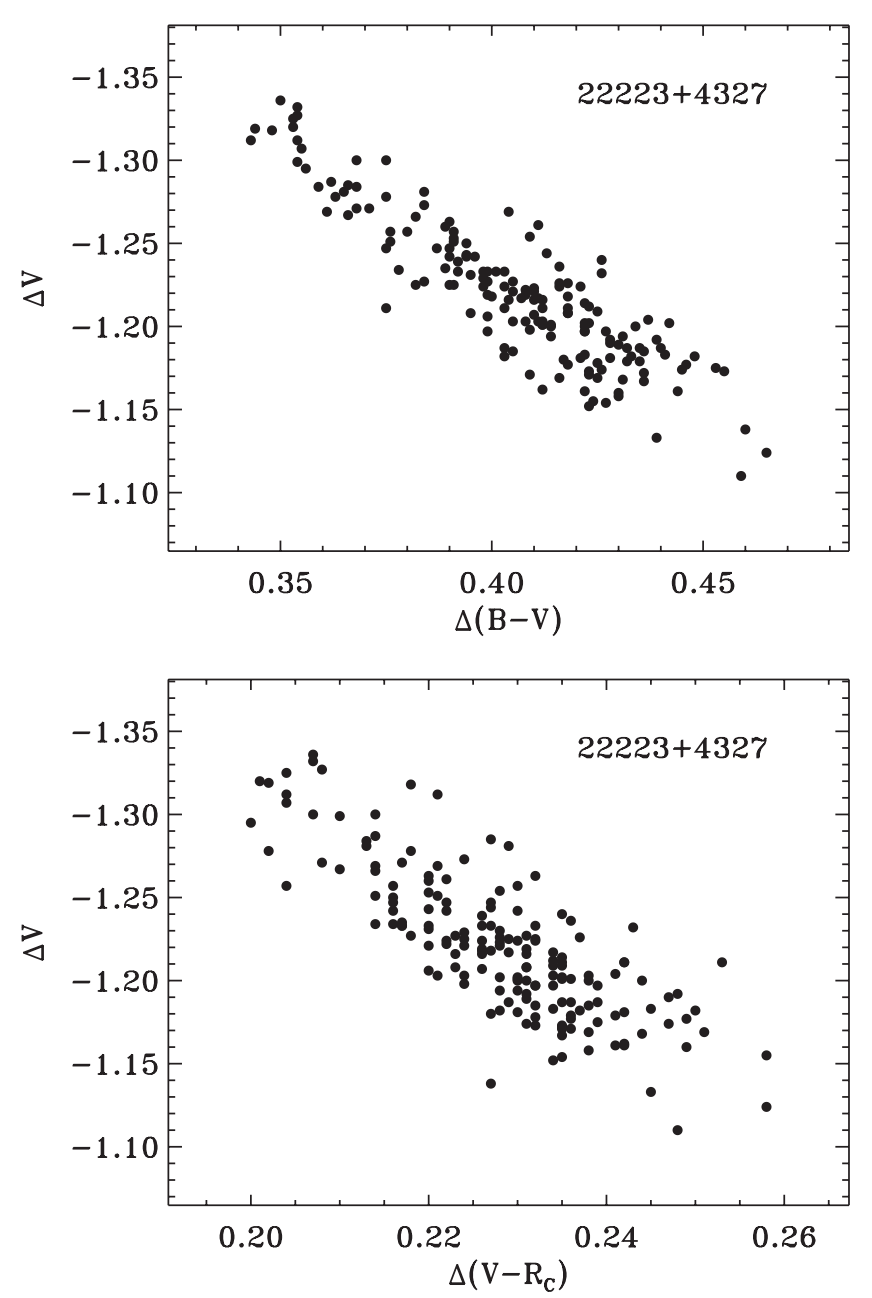

Figure 10. Brightness vs. color curves for our new 2008-2011 observations of IRAS $22223+4327$. They clearly show the correlation of color with brightness; the object is redder when fainter.
2010, consisting of 134 observations in each filter, respectively. They also find a cyclical variation, with a maximum range of $0.25 \mathrm{mag}(V)$, and period values of $85.5 \pm 1$ days and $90.9 \pm 1$ days. Their observations also show the object to be redder when fainter.

Our $V$ observations (1994-2011) and those of Arkhipova et al. $(2003,2011)$ were combined to study the period and light curve variations. The two sets nicely complement each other and fill in the gap in the Paper I $V$ light curve from 2000 to 2002. Our $V$ observations are listed as differential magnitudes and were combined with the standard magnitude of the comparison star $\left(V\left(\mathrm{C}_{1}\right)=11.08\right)$ to give the $V$ light curve. When combining the two data sets, we investigated and found a small offset in brightness, with the Arkhipova et al. $(2003,2011)$ data set brighter in the regions of overlap by 0.019 mag. ${ }^{10}$ For the purposes of this period and light curve study, we have added an offset of +0.020 mag to each of the $V$ magnitudes of Arkhipova et al. (2003, 2011). The resulting combined $V$ light curve is shown in Figure 11.

This combined $V$ light curve of IRAS $22223+4327$ covers the interval 1994-2011. As noted earlier, the light curves show a general decrease in brightness. From 1994 to 2002, the $V$ light curve decreased by $0.06 \mathrm{mag}$. The decrease was more rapid from 2002 to $2008,0.09 \mathrm{mag}$ or $\sim 0.015 \mathrm{mag} \mathrm{yr}^{-1}$. From 2008 to 2011 the average light levels are approximately constant, indicating a leveling off of the decline. This is also seen in our combined $R_{C}$ light curve from 1995 to 2011. We suggested earlier (Hrivnak et al. 2010) that this might be due to an increase in line-of-sight dust extinction during this time. However, the $\left(V-R_{C}\right)$ color is constant over the entire interval of 1995-2011, with no evidence

${ }^{10}$ Note that the offset is based on 48 measurements of the difference between our standard magnitudes and those of Arkhipova et al. (2003, 2011), observed on the same night or with a difference one day between them. This difference can be compared with the internal difference within our own data set, where we have observations on 49 nights in which we have data observed one night apart, and for which we found a difference of $0.002 \mathrm{mag}$; similar internal comparison of the data of Arkhipova et al. shows a difference of -0.004 mag on 12 nights. 


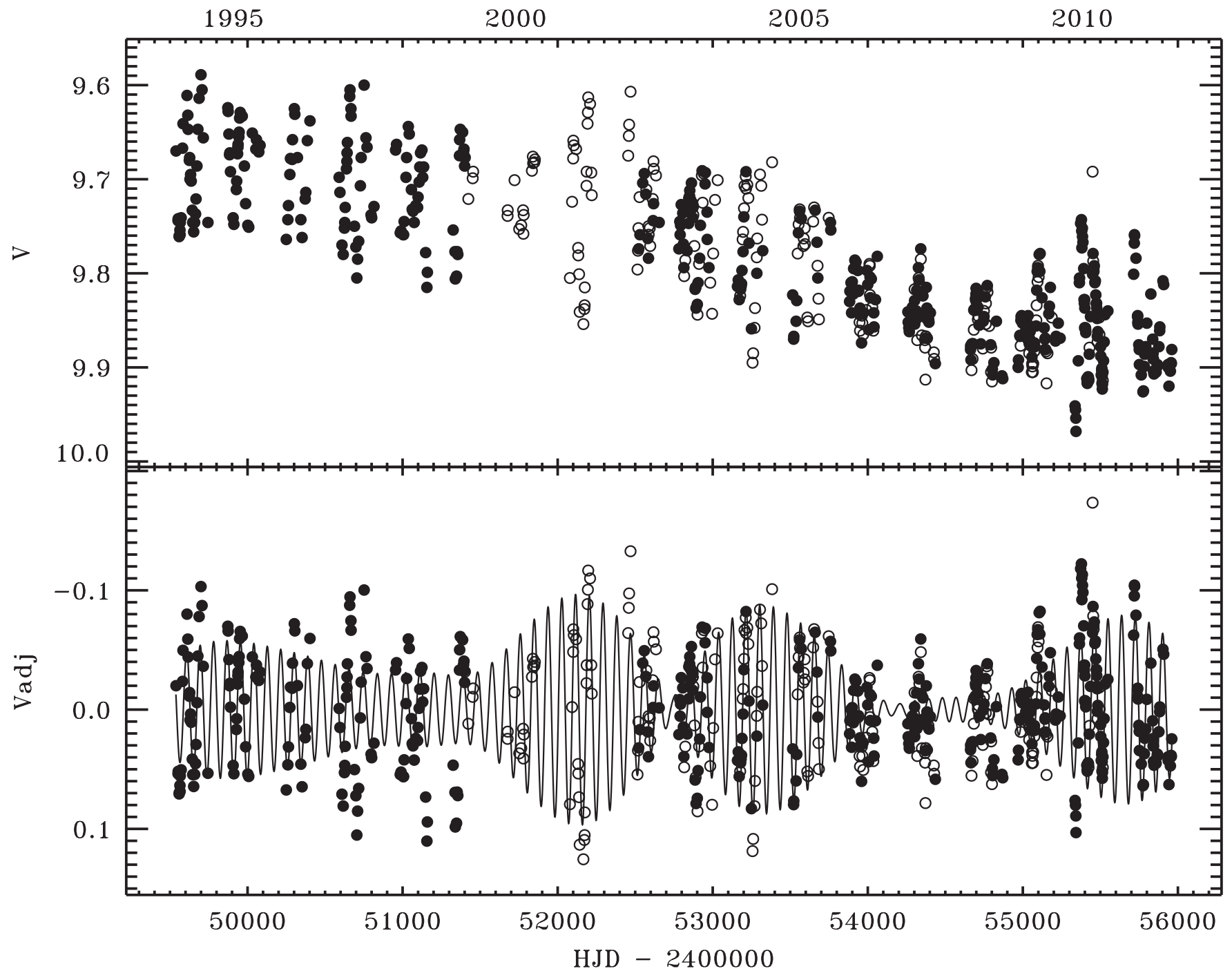

Figure 11. Top: the combined $V$ light curve of IRAS 22223+4327 from 1994 to 2011 . Bottom: the combined $V$ light curve fitted with the first four periods and amplitudes of the periodogram analysis. The general trend of decreasing brightness was first removed using a fourth-order polynomial fit. The filled circles are the data from the VUO and the open circles from Arkhipova et al. $(2003,2011)$.

for increased reddening with decreased brightness; Arkhipova et al. (2011) make similar comments about the constancy of the $(B-V)$ and $(U-B)$ color indices. Thus, if the dimming is due to dust extinction, then the scattering must be gray, which would suggest relatively large $(\geqslant 1 \mu \mathrm{m})$ dust particles.

We have proceeded to carry out a period analysis on the light curves of IRAS $22223+4327$, similar to what we did with IRAS $22272+5435$. In this case, to account for the decrease in brightness, the $V$ and $R_{C}$ light curves were fit with a fourth-order polynomial, which was then used to remove the general monotonic decrease. The relatively large variation in the seasonal amplitudes suggests at least the existence of two periods in the data. The results are as follows and are tabulated in Table 9.

A. The combined V data set from 1994 to 2011. For the combined $V$ data set, there is a dominant period of $90.5 \pm$ 0.1 days with a second period of $85.8 \pm 0.1$ days. These values are very similar to those found by Arkhipova et al. (2011), although we find the larger of the two to be the clearly dominant one. The ratio of the periods is $P_{2} / P_{1}=$ 0.95 , the same as found for IRAS $22272+5435$. The power spectrum of the first period is shown in Figure 12, along with a phase diagram from this single period. Comparing the Fourier components of the fit with two periods to the light curve results in reasonably good agreement, and this is improved with a fit to four periods, which is shown in the bottom panel of Figure 11. While the constructive interference of several curves with different periods helps to fit the large amplitude seasons, the fit is poor, however, where the curves interfere destructively, such as in 2008 and 2009.

B. The combined V data set from 1994 to 2002. The combined $V$ data set from the first nine years yields values that are similar to the periods determined from the entire combined $V$ light curve, with $P_{1}, P_{2}$, and $P_{4}$ slightly smaller and $P_{3}$ slightly larger. The first period is noticeably dominant in this data set; $P_{4}$ is barely significant.

C. The combined $V$ data set from 2003 to 2011. The $V$ data set from the last nine years yields $P_{1}=86.7 \pm 0.1$ days, a value that is similar to $P_{2}$ of the combined data set, and $P_{2}=89.5 \pm 0.1$ days, a value that is similar $P_{1}$ of the combined data set (differing at the $1 \%$ level). These are similar to the values found by Arkhipova et al. (2011) for their 2003-2010 data. The amplitudes of $P_{1}$ and $P_{2}$ in the 
Table 9

Periodogram Study of the Light and Color Curves of IRAS $22223+4327^{\text {a }}$

\begin{tabular}{|c|c|c|c|c|c|c|c|c|c|c|c|c|c|c|c|}
\hline $\begin{array}{l}\text { Data } \\
\text { Set }\end{array}$ & Filter & Years & $\begin{array}{l}\text { No. } \\
\text { Obs. }\end{array}$ & $\begin{array}{c}P_{1} \\
\text { (days) }\end{array}$ & $\begin{array}{c}A_{1} \\
(\mathrm{mag})\end{array}$ & $\phi_{1}{ }^{\mathrm{b}}$ & $\begin{array}{c}P_{2} \\
\text { (days) }\end{array}$ & $\begin{array}{c}A_{2} \\
(\mathrm{mag})\end{array}$ & $\phi_{2}{ }^{\mathrm{b}}$ & $\begin{array}{c}P_{3} \\
\text { (days) }\end{array}$ & $\begin{array}{c}A_{3} \\
(\mathrm{mag})\end{array}$ & $\phi_{3}{ }^{b}$ & $\begin{array}{c}P_{4} \\
\text { (days) }\end{array}$ & $\begin{array}{c}A_{4} \\
(\mathrm{mag})\end{array}$ & $\phi_{4}{ }^{\mathrm{b}}$ \\
\hline A & $V$ & 1994-2011 & 630 & 90.5 & 0.040 & 0.24 & 85.8 & 0.029 & 0.89 & 92.4 & 0.020 & 0.22 & 83.8 & 0.016 & 0.55 \\
\hline B & $V$ & 1994-2002 & 210 & 89.7 & 0.052 & 0.00 & 83.3 & 0.027 & 0.17 & 96.2 & 0.021 & 0.06 & 81.0 & 0.018 & 0.86 \\
\hline $\mathrm{C}$ & $V$ & 2003-2011 & 420 & 86.7 & 0.033 & 0.61 & 89.5 & 0.028 & 0.65 & 94.3 & 0.014 & 0.56 & $\ldots$ & $\ldots$ & $\ldots$ \\
\hline $\mathrm{D}$ & $R_{C}$ & $2003-2011$ & 289 & 86.6 & 0.022 & 0.58 & 89.9 & 0.019 & 0.80 & $\ldots$ & $\ldots$ & $\ldots$ & $\ldots$ & $\ldots$ & $\ldots$ \\
\hline $\mathrm{E}$ & $R_{C}$ & 1995-2011 & 387 & 90.5 & 0.026 & 0.24 & 85.7 & 0.018 & 0.86 & 67.8 & 0.014 & 0.90 & 74.3 & 0.012 & 0.49 \\
\hline $\mathrm{F}$ & $V-R_{C}$ & 1995-2011 & 332 & 90.4 & 0.010 & 0.16 & 86.1 & 0.007 & 0.04 & 91.9 & 0.007 & 0.89 & 78.8 & 0.004 & 0.93 \\
\hline
\end{tabular}

Notes.

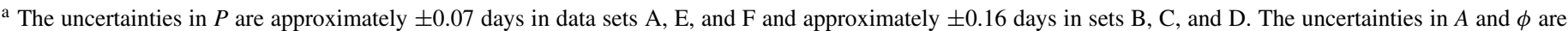

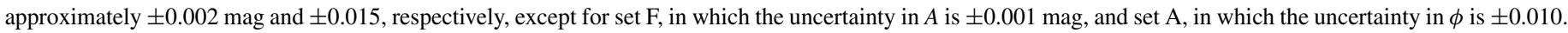

$\mathrm{b}$ The phases are determined based on the epoch of 2,448,000.00.
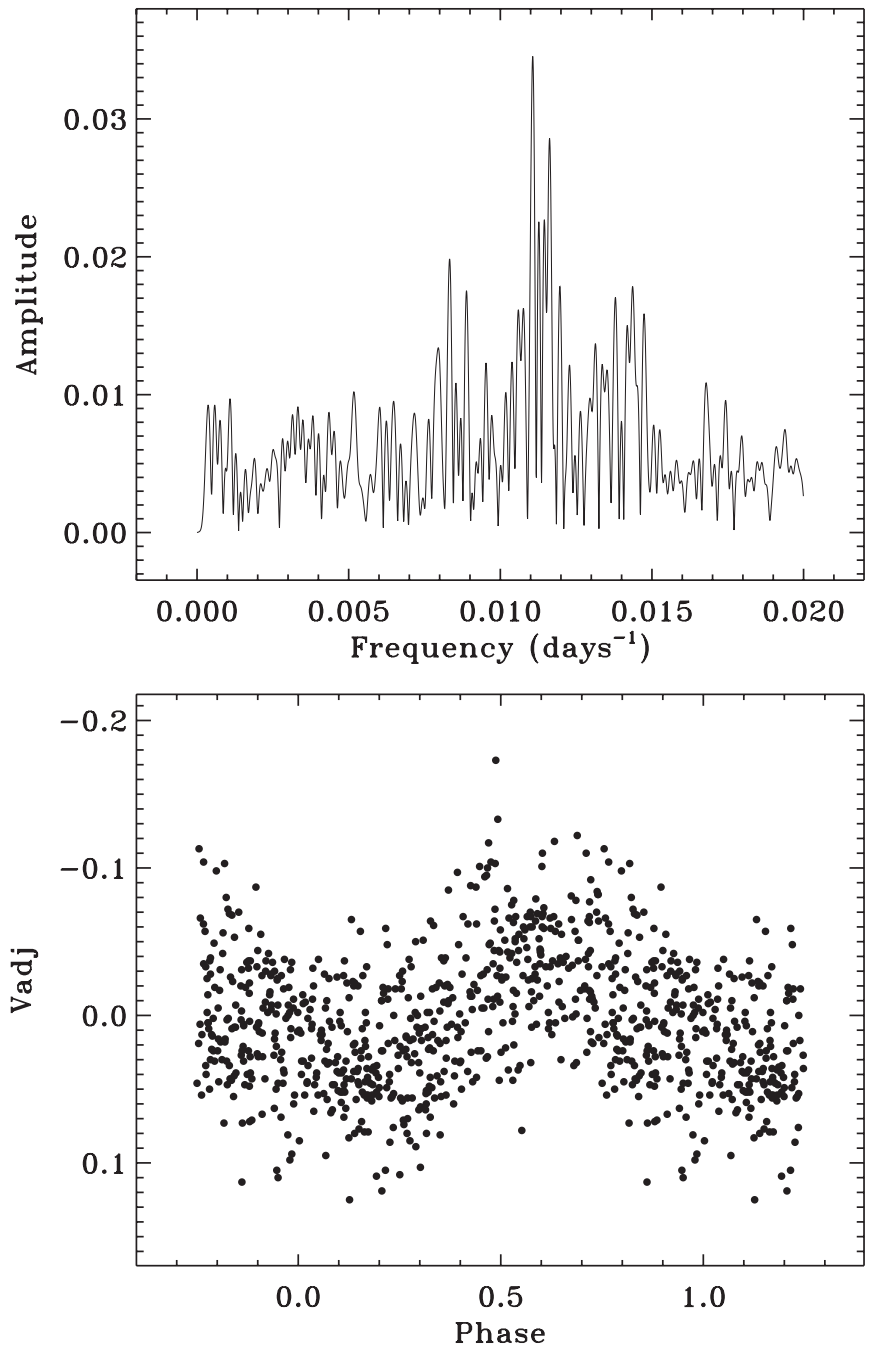

Figure 12. Top: the frequency spectrum for the first period of the $V$ light curve of IRAS 22223+4327. Bottom: the phase plot of the $V$ light curve based on the frequency peak of $P_{1}=90.5$ days. These both use the adjusted data following the removal the long-term trend by a fourth-order polynomial fit.

2003-2011 data set are relatively similar, and neither one is dominant, in contrast to the 1994-2002 $V$ results. There is no significant $P_{4}$ in this data set.

D. The $R_{C}$ data set of the VUO from 2003 to 2011. The $R_{C}$ data set from the last nine years was also investigated. It yields values for $P_{1}$ and $P_{2}$ that are very similar to those found from the corresponding 2003-2011 $\mathrm{V}$ data set, but no significant $P_{3}$ or $P_{4}$ was found. As noted in the overall light curves, the amplitudes in $R$ are smaller than those in $V$.

E. The $R_{C}$ data set of the VUO from 1995 to 2011 . The entire $R_{C}$ data set was analyzed and yielded values for $P_{1}$ and $P_{2}$ that are the same as those found from the entire $V$ data set and similar to those found for $P_{2}$ and $P_{1}$, respectively, in the 2003-2011 $R_{C}$ data set. The values for $P_{3}$ and $P_{4}$ differ from those found for the $V$ light curve, with $P_{4}$ barely significant.

F. The $V-R_{C}$ data set of the VUO from 1995 to 2011 . The observed $V-R_{C}$ data set from the VUO from 1995 to 2011 , which lacks data from 2000 and 2001, was analyzed for periodicity. Since the mean $V-R_{C}$ values are similar from season to season, no normalization of the data was carried out. Although the full range in color is small, $0.06 \mathrm{mag}$, nevertheless clear periods were found. The values of $P_{1}$ and $P_{2}$ are very similar to those of the entire $V$ and $R_{C}$ data sets, and $P_{3}$ is similar to that of the entire $V$ data set.

We investigated the evidence for a secular period change by comparing the primary period values of the 1994-2002 data with those of the 2003-2011 data, especially for the $V$ data, which has the larger amplitude of variation. From 1994 to 2002 , the primary period from the $V$ light curve is 89.7 days, while for 2003-2011, there are two periods of relatively similar amplitudes of 86.7 and 89.5 days. The $R_{C}$ light curve from 2003 to 2011 has similar values of $P_{1}$ and $P_{2}$. Thus the results of the period study of these light curves give evidence of multiple periods with some change, but do not give evidence of a secular change in this limited time interval of 18 years of observations.

As with IRAS $22272+5435$, we also investigated the period change with a plot of cycle number versus HJD for the minima of the variability. This is shown in Figure 13, based on visual inspection of the VUO $V$ and $R_{C}$ light curves. On this scale it looks linear, with a slope yielding a period of 87.1 days, a value about halfway between the two dominant periods found in the periodogram analysis. The residuals show a systematic behavior, which could be interpreted as a relatively constant period with a slight increase $(E=0-15)$, followed by a large increase in period $(E=15-30)$, then a sudden decrease in period $(E=35-37)$, and finally a relatively constant period with a slight decrease $(E=37-68)$. Note that the full range of the residuals is almost as large as the period. The pattern does not repeat during the 18 years of our observations. As with IRAS 22272+5435, we examined the residuals from the theoretical light curve based on the four periods and corresponding amplitudes, and, as with 


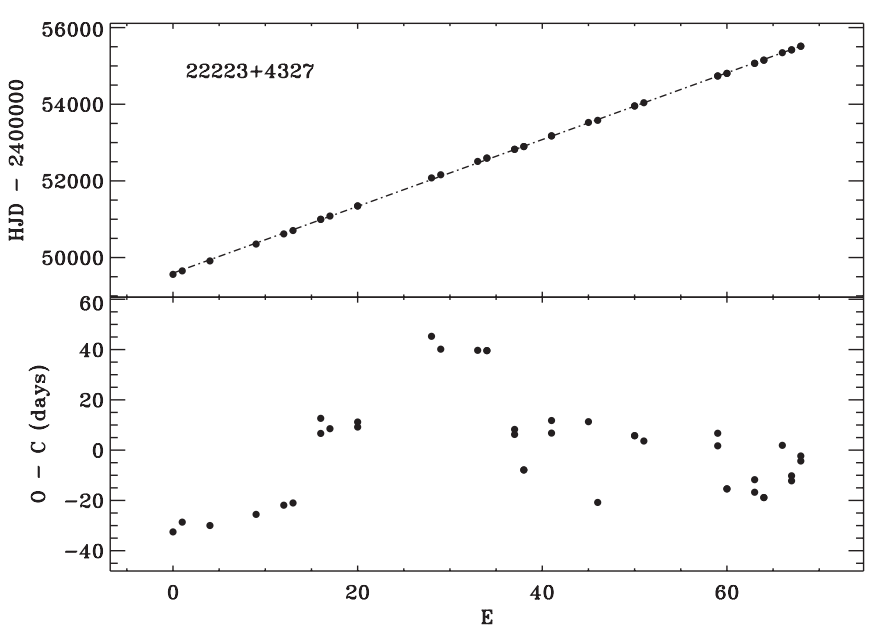

Figure 13. Top: observed time of minimum vs. cycle count for the $V$ and $R_{C}$ light curves of IRAS $22223+4327$. The slope of the line is 87.1 days cycle ${ }^{-1}$. Bottom: the residuals of the time of minimum about the straight-line fit.

IRAS $22272+5435$, they had a similar range in $(O-C)$ values but not the same pattern.

\subsection{Radial Velocity Study}

For the radial velocity study of IRAS $22223+4327$, we combined our DAO-RVS observations from 1991 to 1995 with our more recent observations from DAO-CCD (2007-2011), CORAVEL (2008-2011), and Hermes (2009-2011). Based on our experience in combining our three recent sets of radial velocity measurements for IRAS $22272+5435$, we also inter-compared the measurement for IRAS 22223+4327. For
Table 10

Periodogram Study of the Radial Velocity Curve of IRAS $22223+4327^{a}$

\begin{tabular}{lccccccccc}
\hline \hline $\begin{array}{l}\text { Data } \\
\text { Set }\end{array}$ & Years & $\begin{array}{r}\text { No. } \\
\text { Obs. }\end{array}$ & $\begin{array}{c}A_{0} \\
\left(\mathrm{~km} \mathrm{~s}^{-1}\right)\end{array}$ & $\begin{array}{c}P_{1} \\
(\text { days })\end{array}$ & $\begin{array}{c}A_{1} \\
\left(\mathrm{~km} \mathrm{~s}^{-1}\right)\end{array}$ & $\begin{array}{c}\phi_{1}{ }^{\mathrm{b}} \\
P_{2} \\
(\text { days })\end{array}$ & $\begin{array}{c}A_{2} \\
(\mathrm{mag})\end{array}$ & $\phi_{2}{ }^{\mathrm{b}}$ \\
\hline A & $1991-2011$ & 189 & -41.10 & 88.8 & 1.95 & 0.49 & $\ldots$ & $\ldots$ & $\ldots$ \\
B & $2007-2011$ & 147 & -41.20 & 88.3 & 1.99 & 0.96 & $\ldots$ & $\ldots$ & $\ldots$ \\
C & $1991-1995$ & 34 & -40.58 & $88.8^{\mathrm{c}}$ & 2.20 & 0.50 & $\ldots$ & $\ldots$ & $\ldots$ \\
\hline
\end{tabular}

Notes.

a The uncertainties in $P$ vary quite a bit depending upon the length of the observing interval, and are approximately \pm 0.05 days (set A), \pm 0.3 days (set B), and \pm 0.5 days (set C). The uncertainties in $A$ and $\phi$ are much more similar among the sets, and are approximately $\pm 0.20 \mathrm{~km} \mathrm{~s}^{-1}$ and \pm 0.02 , respectively.

$\mathrm{b}$ The phases are determined based on the epoch of 2,448,000.00.

c This period determination does not quite reach our accepted level of significance, but is included to show the agreement with the other values.

IRAS $22223+4327$, the data are fewer and the results are less certain. There may be a zero-point shift of approximately $+0.5 \mathrm{~km} \mathrm{~s}^{-1}$ in the CORAVEL and perhaps about half that value in the Hermes measurements as compared with the DAO-CCD measurements, but this is rather uncertain and so no offset was applied. Thus we combine the data as measured, including the DAO-RVS. In addition, there exist two published velocities by Van Winckel \& Reyniers (2000) from 1994 and nine by Klochkova et al. (2010) from 1998 to 2008, and we have also included them in the velocity analysis. The combined radial velocity curve is shown in Figure 14.

We discuss below the results of the analysis of the combined radial velocity measurements and for the data in subsets from 1991-1995 and 2007-2011. The results are listed in Table 10.
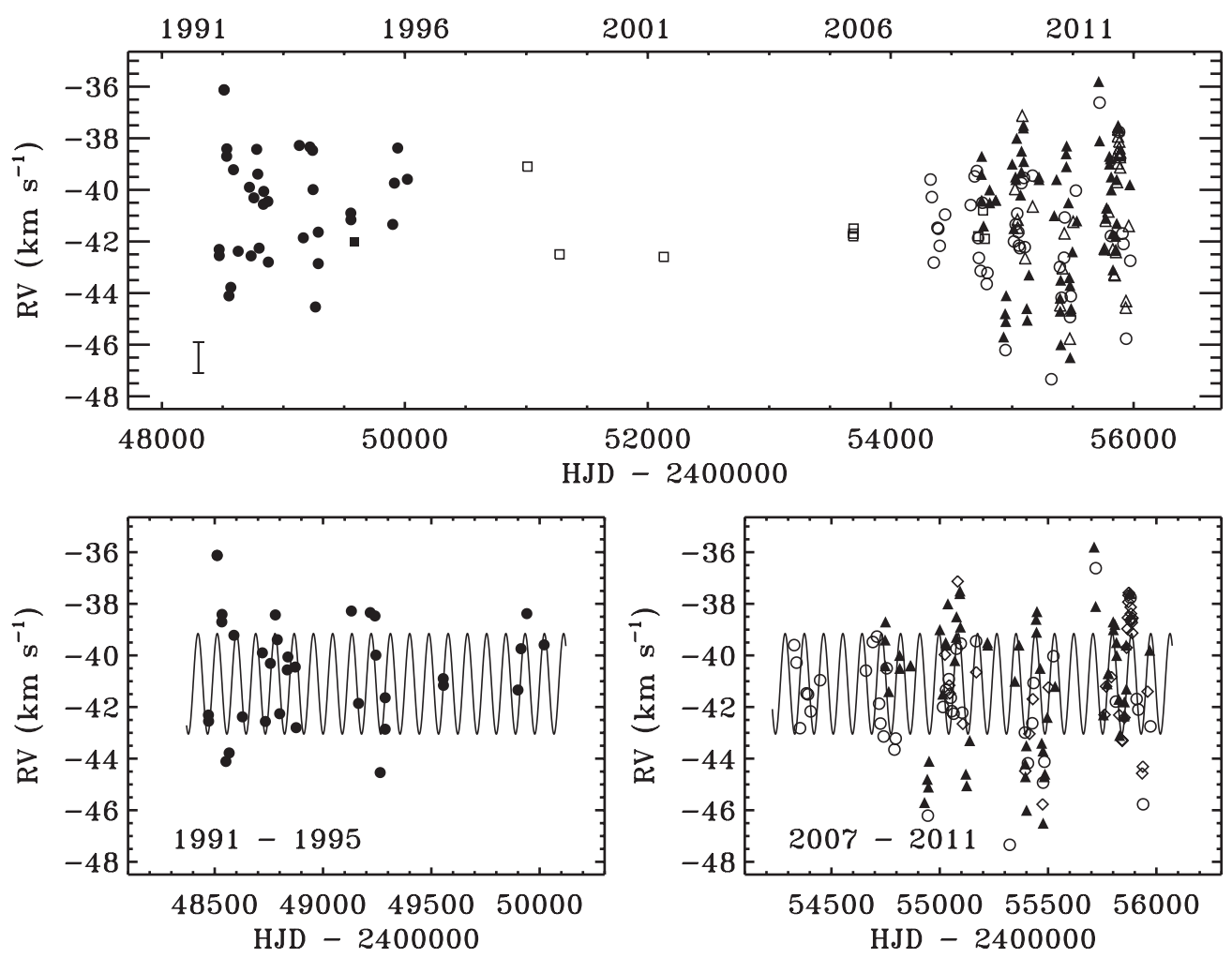

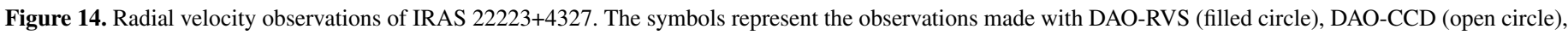

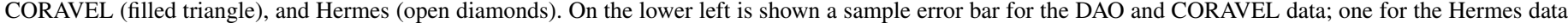

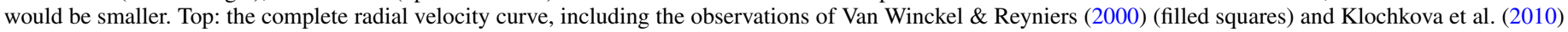
(open squares). Bottom: sine-curve fits to the velocities based on $P=88.8$ days, displayed over shorter intervals of time, 1991-1995 and 2007-2011. 

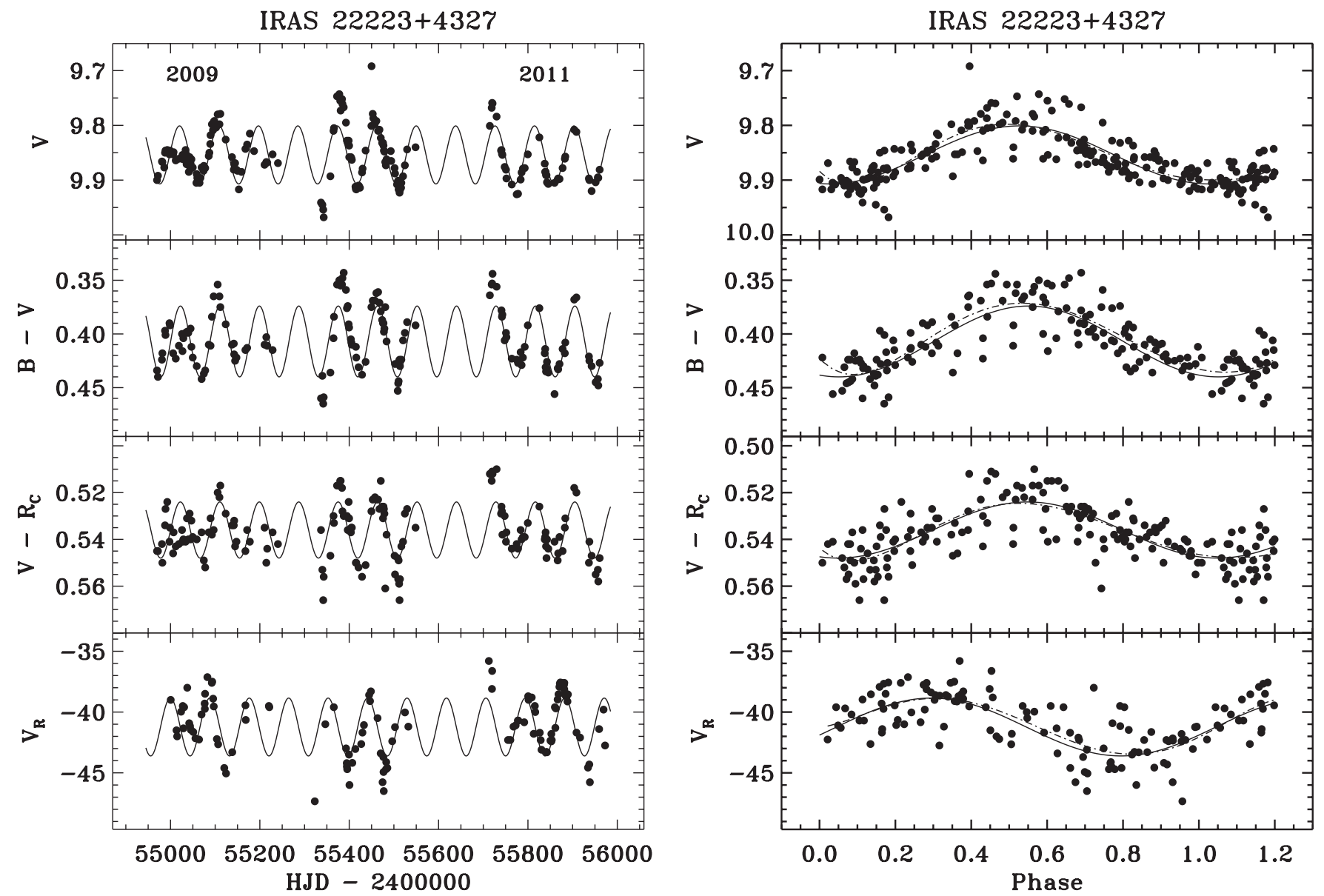

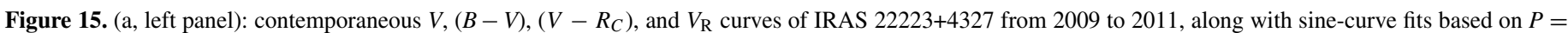

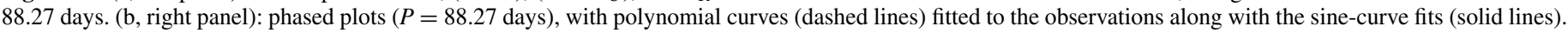

A. The combined radial velocity data set from 1991 to 2011. The data show one dominant period in the data set, $P_{1}=$ $88.8 \pm 0.1$ days. This value of $P_{1}$ is close to the average value of the two main periods determined from the light curves. A second period of 167.3 days is found in the velocities but not quite at the level to be regarded as significant. The value of $P_{1}$ and the associated amplitude and phase give a reasonably good fit to the observed velocities, as shown in Figure 14. One can see that at some times the velocity amplitude is not large enough. Including the second period mentioned above would help somewhat to improve this.

B. The combined radial velocity data set from 2007 to 2011. This is a reasonably large data set (145 points) and possesses a dominant period of $P_{1}=88.3 \pm 0.3$ days, close to the value found for the entire radial velocity data set. It gives a good fit to these data.

C. The combined radial velocity data set from 1991 to 1995. This is a small data set of 34 observations spread over five seasons. They yield a period of 88.9 days, although at an $\mathrm{S} / \mathrm{N}$ that does not quite reach our accepted level of significance. This gives a good fit to the 1991-1995 velocities and the period is in good agreement with that found for the entire data set.

Thus a single dominant period of 88.8 days is found from the radial velocity data, and this value is an approximate (weighted) mean of the two dominant periods found in the light curve data.
The velocity curve can also be fitted reasonably well using the two dominant periods found in the light curve.

\subsection{Contemporaneous Light, Color, and Velocity Curve Study}

Contemporaneous light, color, and velocity curves exist for IRAS 22223+4327 from 2007 through 2011. We decided to use for comparison the 2009-2011 observations, since for these three seasons the observations are relatively similar. We did not use the 2007 data since there are only six radial velocity observations, and we did not use the 2008 data since the light, color, and velocity amplitudes were significantly smaller than in the following three years. For the light and color curves we used only the VUO data; the light and color curves are well defined by these observations and this helps assure a more homogeneous data set.

These observations are shown in Figure 15(a). Fitted to these are sine curves based on $P=88.27$ days (deduced from the 2009-2011 $V$ light curve) and the arbitrary epoch of 2,448,000. The curves are fitted with semi-amplitudes and phases, respectively, as follows $-V$ : $0.053 \mathrm{mag}$ and $0.22, B-V$ : $0.033 \mathrm{mag}$ and $0.20, V-R_{C}: 0.012 \mathrm{mag}$ and 0.20 , and $V_{\mathrm{R}}$ : $2.38 \mathrm{~km} \mathrm{~s}^{-1}$ and 0.96 . Thus the light and color curves are very nearly in phase, with the color reaching maximum blueness 0.02 in phase earlier than maximum light, and the radial velocity differs by almost a quarter of a cycle $(0.27)$. The uncertainties in the phases are $\pm 0.01-0.02$. The fit of the $V, B-V$, and even the low-amplitude $V-R_{C}$ curves is quite good, and the fit of the $V_{\mathrm{R}}$ curve is reasonable. Shown in Figure 15(b) are the 
observations phased to this period and epoch. The observed range in amplitude shows up as vertical scatter in the panels. Both the sine-curve fits and fourth-order polynomial fits are shown for the phased data. The polynomials do not differ much from the sine curves. The slight phase difference between the $B-V$ and $V$ curves shows up, and the $V_{\mathrm{R}}$ curve appears to have a somewhat more gradual decline and somewhat steeper rise than the sine curve. As with the 2005-2007 data of IRAS $22272+5435$, the sine curves can be seen to produce good fits to the observations of IRAS 22223+4327 during 2009-2011.

\section{DISCUSSION}

\subsection{Period Study}

The light curves of both PPNs show evidence of multiple periods that modulate the light curves. This appears to not be uncommon, as Kiss et al. (2007) found evidence for this among several other post-AGB stars. Four significant periods are found in the light curves of each of these two PPNs, and they can be fit reasonably well with a series of four sine curves. For the radial velocity curves, which have fewer data points, one to three sine curves give reasonably good fits. IRAS $22272+5435$ shows excellent agreement between the values of $P_{1}$ and $P_{2}$ found for the light curves and velocity curves, with differences less than $1 \%$. For IRAS $22223+4327$, only one period was determined from the radial velocity curve ( 88.8 day) and it fell between the values of $P_{1}$ (90.5 day) and $P_{2}(85.8$ day) found from the light curve.

For both of the PPNs, we find from the complete $V$ light curves a ratio of pulsation periods, $P_{2} / P_{1}$, of 0.95 . Similar values are found by Arkhipova et al. (2010, 2011) for two PPNs (0.92, 0.96), by us for several O-rich PPNs (B. J. Hrivnak et al. 2013, in preparation, Paper III), by Kiss et al. (2007) for three postAGB stars (0.90-0.94), and by Van Winckel et al. (2009) for two post-AGB RV Tau stars $(0.91,0.96)$. It appears that two closely spaced periods give a good fit to the modulated light curves of these post-AGB objects and may be a characteristic feature of their pulsational variability. These are different from the typical ratios between the first overtone and the fundamental modes of $0.71-0.73$ found for double-mode classical Cepheids in the Milky Way and the Magellanic Clouds (Beaulieu et al. 1997). Note that the double-mode Cepheids have periods of only 2-6 days and lie near the blue edge of the instability strip. This difference in pulsation ratio may reflect the differences in the mass or internal structure between these low-mass post-AGB stars and the more massive Cepheids.

We also investigated the data for evidence of changes in the light curve periods in the $\sim 20$ years of observations. Hrivnak et al. (2010) suggested that there may be period decreases of $\sim 2$ days over 14 years based on the period-temperature relationships that they measured and the model calculations of Steffen et al. (1998) for the evolution of a carbon-rich central star of $0.605 M_{\odot}$. However, we have not found this. For IRAS $22272+5435$, exactly the same value of the primary period $P_{1}$ was found in the light curves from 1991 to 1999 and from 2002 to 2011 (although the values of $P_{2}$ differed). For IRAS 22223+4327, there appears to be some change in the periods, with the light curve from 1994 to 2002 having dominant periods of $P_{1}=89.7$ and $P_{2}=83.3$ days, while the light curve from 2003 to 2011 has two periods of more similar strength with $P_{1}=86.7$ and $P_{2}=89.5$ days. However, this small change is not easy to interpret and does not appear to indicate a shortening of the period. Also, monotonic period changes are not seen in the plots of the residuals from a linear fit to the observed times of minimum light versus cycle count (Figures 5 and 13). Such evidence needs to be investigated carefully for more PPNs and likely over longer temporal baselines before one can make a stronger assertion regarding a secular period change.

\subsection{Contemporaneous Light, Color, and Velocity Curves}

The contemporaneous light, color, and velocity curves provide an opportunity to study the pulsation in more detail. For IRAS 22272+5435, the observations from 2005 to 2007 appear to be reasonably similar, as shown in Figure 7 . The $V, V-R_{C}$, and radial velocity curves are well represented by sine curves using the parameters listed in Section 5.3. They show that the light and color curves have the same phasing and that the radial velocity curve differs in phase by -0.26 , almost exactly a quarter of a cycle. This indicates that the star is brightest when hottest and smallest. Polynomial fits to the data are also shown. For the radial velocity curves, the two different fits are very similar. For the light and color curves, the polynomial fits have similar shapes to each other and both differ slightly from the sine fits. The polynomial fits reach maximum a little earlier and minimum a little later in phase, resulting in a slightly slower decline to minimum and a steeper rise to maximum. Contemporaneous observations for IRAS $22272+5435$ also exist from 2008 to 2011, and we have examined the more similar years 2008-2009. While these data are not so well fitted by sine curves, they do indicate that on average the light and color are nearly in phase and the radial velocity curve again about a quarter of a cycle $(-0.22)$ different. A similar comparison can be made for the contemporaneous observations of IRAS 22223+4327, and we have done so using the data from 2009 to 2011. One again finds that the light and color curves are nearly in phase (differing by -0.02 ) and the radial velocity differs by about a quarter of a cycle $(-0.27)$. Again, comparison with polynomial fits shows that sine curves are a good representation of the variations. Overall, given the intrinsic changes in the variability from season to season and even over a single season, to within the uncertainty one can state that the curves are all well represented by sine curves, with the light and color curves in phase and the radial velocity curve differing in phase by a quarter of a cycle $(-0.25)$.

\subsection{Comparison with Other Evolved Pulsating Stars}

These observed curves are quite different from those of classical Cepheids pulsators in the following ways: (1) in their shapes-in Cepheids the light and color curves show a steeper rise and a more gradual decline, with the velocity curves showing the opposite; (2) in the phasing between the light and color curves-in Cepheids the color minimum is later than the light minimum; (3) in the phasing of the light and velocity curve-in Cepheids they are the mirror images of each other, differing in phase by 0.50 . Thus in Cepheids the photosphere is moving toward the observer at maximum speed and at approximately average size when it is at light maximum, while in these PPNs the photosphere is temporarily stationary at a minimum size at light maximum. In other words, these two PPNs do not display the phase lag seen in classical Cepheids, which is caused by the delaying action of the hydrogen partial ionization zone on the energy flux (Ostlie \& Carroll 2007).

RV Tauri variables have more similar masses and might be a better comparison. They have mid-F-K spectral types and spectra of luminosity classes I-II. Their light curves are characterized by alternating deeper and shallower minima, with 
periods between deeper minima of 50-150 day (Wahlgren 1993). Strong Balmer line emission and metallic line doubling is observed and attributed to shocks. A comparison of the light, color, and velocity curves is complicated by the variation in these curves from cycle to cycle and by the lack of contemporaneous light and velocity curves. McLaughlin (1941), in an old study, attempted to define from observations a generalized light and velocity curve for an "ideal RV Tauri variable." He concluded that the velocity maximum occurs a few days after the deeper light minimum. In his associated figure (Figure 6), it appears that the deeper minimum occurs when the star is close to, but slightly after, largest size and that the brighter maximum occurs when the star is close to, but slightly before, minimum size. This is more similar to what we see in these two PPNs. However, there is a significant difference in that the light and velocity curves of RV Tauris display alternating deeper and shallower light and velocity minima that are not characteristic of the PPN light and velocity curves. Pollard et al. (1996, 1997) carried out a comprehensive photometric and spectroscopic study of 11 RV Tauri variables over two to four seasons. These RV Tauri variables showed a much larger range of variation in brightness $(0.8-3.0 \mathrm{mag}$ in $V)$ and radial velocity $\left(15-50 \mathrm{~km} \mathrm{~s}^{-1}\right)$ than is seen in these two or other PPNs (Hrivnak et al. 2010, 2011). They did not have good enough contemporaneous coverage of light and velocity to do an accurate comparison of the phasing, so unfortunately we cannot compare the relative phasing of the RV Tauris with that found for these two PPNs. The color curves do show that for RV Tauris the light is bluest on the rise to maximum light, preceding the light peak by $\sim 0.1$ in phase. Our PPN data may show a slight tendency to be bluer before the light maximum, but only by $\sim 0.02$ in phase, which is at the level of our uncertainty.

\subsection{Determination of Radii and Luminosities}

One can use the observed radial velocity curve to calculate the change in radius of the pulsating star. Since the radial velocity curve is well represented by a sine curve, we have simply used this to calculate the change in radius throughout the pulsation cycle. Integrating the radial velocity curve for IRAS $22272+5435$ with its semi-amplitude of $3.02 \mathrm{~km} \mathrm{~s}^{-1}$ over the period of 131.9 days yields a semi-amplitude for the radius curve of $5.48 \times 10^{6} \mathrm{~km}$, or $7.9 R_{\odot}$. To convert the radial velocity to pulsational velocity requires one to correct for geometric projection, atmospheric effects, and other complications (Barnes 2009). Using the Cepheid equation of Nardetto et al. (2009) based on cross-correlation velocities, $p=$ $1.31( \pm 0.06)-0.08( \pm 0.05) \log P$, gives $p=1.15$. Assuming this value for $p$ yields a total change in radius of $1.26 \times 10^{7} \mathrm{~km}$, or $18.1 R_{\odot}$. This is a very large value. The brightness only changes by $0.34 \mathrm{mag}(V)$ and the color varies by 0.076 in $\left(V-R_{C}\right)$ during this time.

For IRAS $22223+4327$, the radial velocity semi-amplitude of $2.38 \mathrm{~km} \mathrm{~s}^{-1}$ and period of 88.3 days result in a semi-amplitude of the radius curve of $2.89 \times 10^{6} \mathrm{~km}$, or $4.2 R_{\odot}$, and with the above projection factor leads to a total radius change of $9.6 R_{\odot}$. This is coupled with a brightness change of $0.106 \mathrm{mag}(V)$ and color changes of $0.066 \mathrm{mag}$ in $(B-V)$ and $0.024 \mathrm{mag}$ in $\left(V-R_{C}\right)$.

We attempted to use these changes in size, along with changes in brightness and temperature (from color) to determine the linear size and luminosity of the stars. Assuming that the temperature determined in the abundance analyses listed in Table 1 is an average temperature and assuming a color-temperature relationship for PPNs similar to that of supergiants, which is a reason- able approximation given the $\log g$ values for these two PPNs of 0.5 and 1.0 , one finds that the color range of $\Delta\left(V-R_{C}\right)=$ 0.076 for IRAS $22272+5435\left(T_{\text {eff }}=5750 \mathrm{~K}\right)$ translates to a temperature variation of $\Delta T_{\text {eff }}=476 \mathrm{~K}$ and a variation in bolometric correction of $\Delta(\mathrm{BC})=-0.057$ (Cox 2000). For IRAS $22223+4327\left(T_{\text {eff }}=6500 \mathrm{~K}\right)$, one finds that the color range of $\Delta\left(V-R_{C}\right)=0.024$ translates to $\Delta T_{\text {eff }}=170 \mathrm{~K}$ and $\Delta(\mathrm{BC})=-0.011$. Knowing the difference in $V$ brightness from maximum to minimum and the difference in bolometric correction at these two phases based on the temperature change, one can find the luminosity ratio between maximum and minimum brightness. Given that the stars are brightest and hottest when smallest, and faintest and coolest when largest, and assuming that the $T_{\text {eff }}$ is the average temperature and that the stars behave like blackbodies, we can then find the ratio of minimum to maximum radius. Knowing the ratio and the difference allows us to then calculate the radius of each of these PPNs. For IRAS $22272+5435$, this leads to a ratio of luminosities of 0.773 , a ratio of radii of 1.038 , and an average radius of $555 R_{\odot}$. This radius value is much larger than that for a post-AGB star of this temperature, and leads to a luminosity of $3.0 \times 10^{5} L_{\odot}$. Such a luminosity is that of a yellow supergiant or hypergiant. It is much larger than that of a post-AGB star, which from theoretical models fit to the luminosities of PN is expected to be in the range of $4-10 \times 10^{3} L_{\odot}$ (Blöcker 1995), and which is determined for LMC carbon-rich PPNs to have a value of 4-10 $\times$ $10^{3} L_{\odot}$ (Volk et al. 2011). Assuming a typical of $L=8 \times 10^{3} L_{\odot}$ leads to a radius of $90 R_{\odot}$ and would require a larger variation in brightness and color (temperature) or a smaller range in radius of $3.4 R_{\odot}$, one-fifth of the value determined from the radial velocity curve. A similar discrepancy occurs for the parameters of IRAS $22223+4327$.

On the other hand, one gets somewhat smaller than expected values for the luminosity and radius when using the spectroscopically determined $T_{\text {eff }}$ and $\log g$ values. These lead to values of $5100 L_{\odot}$ and $72 R_{\odot}$ for IRAS $22272+5435$ and $2600 L_{\odot}$ and $41 R_{\odot}$ for IRAS $22223+4327$ for $M=0.60 M_{\odot}$, with values of luminosity and radius that are larger by 1.33 and 1.15 , respectively, for $M=0.80 M_{\odot}$. Clearly there are unresolved complications in both the pulsational light and velocity curves, and even in the spectroscopically determined physical parameters, that are not presently understood.

The previous spectroscopic study by Začs et al. (2009) had demonstrated the presence of shocks in the pulsating atmosphere of IRAS $22272+5435$. This was revealed by line profile variations and the doubling of low-excitation lines. Earlier observations of changes between absorption and emission in nearinfrared CO lines in IRAS $22272+5435$ and $22223+4327$ can be attributed to sporadic mass loss or pulsation causing shocks (Hrivnak et al. 1994). So it is clear that we are not dealing with static atmospheres in thermal equilibrium, and more complex models are required to understand their behavior and properties. Similar or perhaps even greater complexity and evidence for shocks have previously been found in the related C-rich PPN IRAS 07134+1005 (HD 56126; Barthès et al. 2000). This object is hotter $\left(T_{\text {eff }}=7250 \mathrm{~K}\right.$; Van Winckel \& Reyniers 2000$)$, with a spectral type of $\sim$ F5 I and a dominant period of 35-40 days (Hrivnak et al. 2010; Barthès et al. 2000). It is likely a more evolved analog of the two PPNs in the present study.

We had anticipated trying to use the Baade-Wesselink method to find the radius of each star. This method uses the difference in luminosity in the pulsation curve at two different phases when the color values, and thus the temperatures, are the same to find 
the ratio of radii. But since the color and luminosity curves are in phase and similar in shape (approximately sinusoidal), the luminosities are the same when the colors are the same and thus the radii are the same at those phases and no ratio can be determined. However, given the unresolved complexities that are manifest in the unrealistically large values derived from the amplitudes of the light and velocity curves above, and even the low values derived from the spectroscopic analysis, the prognosis for obtaining reliable radii from the Baade-Wesselink method would be low.

\subsection{Comparison with Pulsational Models}

These observational results can be compared with pulsational models of post-AGB stars of similar temperatures.

Fokin et al. (2001) computed nonlinear, radiative models for post-AGB stars in the temperature range 5600-6000 K and mass of 0.6 and $0.8 M_{\odot}$. Several patterns emerged: the photometric amplitude increased as temperature decreased, the chaotic components decreased as the luminosity decreased, and as the temperature increased, the power in the lower modes increased as the temperature decreased, and the photometric amplitude increased as the mass of the star decreased. They sought to apply these models to the shorter period ( $\sim 37$ days) PPN IRAS 07134+1005 (HD 56126), which is a carbon-rich F star. They had mixed success. Their models agreed with the period and the amplitude of the radial velocity curve, but produced a larger photometric amplitude than observed in this object and had a mass that was too large $\left(0.8 M_{\odot}\right)$ to be consistent with the luminosity $\left(6000-7000 L_{\odot}\right)$ that they determined. Also, the temperature of the models was much lower than the spectroscopically determined value of the effective temperature, 7250 K (Van Winckel \& Reyniers 2000). Their models are of the appropriate temperature for IRAS $22272+5435$, and give a reasonable fit to both the photometric and radial velocity amplitudes. However, their calculated periods ( $<40$ days) are much shorter than the observed period of $\sim 130$ days.

Recently, Aikawa (2010) published the results of a study of pulsation in post-AGB stars of the following range in temperature and gravity: $\log T_{\text {eff }}=3.7-3.9(5000-8000 \mathrm{~K})$ and $\log g=0.0-1.8$, for masses of 0.6 and $0.8 M_{\odot}$. The models assumed radial pulsation, radiative energy transfer, and ignored convection. He ran linear, radial pulsation models and studied the stability from the fundamental up to the fifth overtone mode. For the temperature and gravity of our two stars, the appropriate models generally were found to be stable in the fundamental and first overtone modes and unstable in the higher order (2-5) overtone modes. For the closest model for IRAS $22272+5435$ (for which $\log T_{\text {eff }}=3.76$ ), the $\log T_{\text {eff }}=3.75$ model produced a fundamental period of 62 days (68 days) for $M=0.6 M_{\odot}$ $\left(0.8 M_{\odot}\right)$, with a first overtone period of 26 days (35 days). For IRAS $22223+4327\left(\log T_{\text {eff }}=3.81\right)$, the $\log T_{\text {eff }}=3.80$ model produced a fundamental period of 26 days (25 days) for $M=0.6 M_{\odot}\left(0.8 M_{\odot}\right)$, with a first overtone period of 14 days (14 days). These computed values of the fundamental period are on the order of one-half to one-third of the observed periods and the overtones are correspondingly even shorter. Nonlinear simulations were then run to produce light curves for the unstable modes. These photometric light curves have short periods and low amplitudes, 14 days and $0.20-0.25$ mag peak-to-peak for the best-fit model for IRAS $22272+5435$ and 4 days and 0.06 mag peak-to-peak for the best-fit model for IRAS $22223+4327$.
Thus these published models are in poor agreement with the observed pulsation periods of these two cool post-AGB stars. Clearly additional modeling efforts, ideally including convection, are needed to find agreement with the observations.

\section{SUMMARY AND CONCLUSIONS}

In this paper, we reported on an intensive photometric and radial velocity study of two PPNs, IRAS 22223+4327 and $22272+5435$. Yearly multicolor light curves from 1994 to 2011 are combined with radial velocities from the intervals 1991 to 1995 and 2005 or 2007 to 2011. The primary results of this study are listed below.

1. The light curves display evidence of multiple periods that modulate the light curves. Each contained four significant periods and the radial velocity curves, which are not based on as extensive data sets, contained one period (IRAS 22223+4327) or three periods (IRAS 22272+5435). The periods determined from the radial velocity measurement agree with those determined from the light curves.

2. The dominant primary periods of the two PPNs are 90.5 days for IRAS $22223+4327$ and 131.9 days for IRAS $22272+5435$. The ratio of secondary to primary periods, based on the light curves, is 0.95 for each of the two. This value has also been found for several other post-AGB stars and may be a characteristic of post-AGB pulsational variability. It is much different from the ratio of $\sim 0.72$ found for Cepheid variables.

3. Comparing contemporary light, color, and radial velocity curves for several years reveals that the light and color curves are in phase, with the objects cooler when fainter and no significant phase lag between the two. The radial velocity curves are $0.25 P$ out of phase with the light curve, with the objects smallest when hottest. The semiamplitudes $\Delta V$ and $\Delta V_{r}$ are small, $0.11 \mathrm{mag}$ and $2.4 \mathrm{~km} \mathrm{~s}^{-1}$ for IRAS $22223+4327$ and $0.34 \mathrm{mag}$ and $3.0 \mathrm{~km} \mathrm{~s}^{-1}$ for IRAS $22272+5435$ for the contemporaneous curves, with shapes that are close to sinusoidal. These are in contrast to Cepheid variables, in which there is a slight phase lag between the color and the light curves and in which the radial velocity curves differ by $0.5 P$ from the light curves, with the Cepheids having average size and expanding when the stars are at maximum brightness.

4. An attempt to use the contemporaneous curves to determine the luminosity and radii of the PPNs led to values that are much too large for PPNs. The source of these discrepancies requires further investigation.

5. These values for the observed periods are much longer than those calculated by nonlinear pulsation models and the observed amplitudes are generally somewhat larger. These point to the need for new models, at least in some cases including convection, to try to understand the pulsational nature of these post-AGB stars that are in transition from the AGB to the PN phases. Such models have the potential to allow the determination of the mass and luminosity of these objects, which presently have no direct means of being determined.

This combination of light, color, and radial velocity observations, especially when they are made contemporaneously, allows one to better constrain the pulsational properties of these stars. The fact that the light curves are complicated, with changing amplitudes and changing spacing between minima, makes it necessary that such long temporal data sets be obtained to sort 
out the component periods and amplitudes. This study is a first step in that direction, and we are in the process of carrying out similar studies of other bright PPNs.

We gratefully acknowledge helpful conversations with P. Lenz regarding Period04 and with D. Turner about variable stars in general. The comments of the anonymous referee were appreciated. We thank R. D. McClure and C. D. Scarfe for making some observations for us, K. L. Wefel who assisted in the reduction of the early DAO radial velocity data, and the many Valparaiso University undergraduate students, most recently Kristie Shaw, Wesley Cheek, Rachael Jensema, Ryan McGuire, Christopher Miko, Zachary Nault, Joel Rogers, Samuel Schaub, and Christopher Wagner, who carried out the photometric observations over these past 18 years. We acknowledge support for this collaboration from the EU FP7-PEOPLE-2010-IRSES program in the framework of project POSTAGBinGALAXIES (grant agreement No. 269193). B.J.H. acknowledges the support of a University Research Professorship and a sabbatical leave from Valparaiso University and the hospitality of the Dominion Astrophysical Observatory during the initial stages of this research project. B.J.H. also acknowledges support from the National Science Foundation (AST 9018032, 9315107, 9900846, 0407087, 1009974), NASA through the JOVE program, and the Indiana Space Grant Consortium. L.Z. acknowledges support from the Latvian Council of Science under grant No. 09.6190, and J.S. and L.Z. acknowledge support from the Research Council of Lithuania under the grant MIP-085/2012. This research has made use of the SIMBAD database, operated at CDS, Strasbourg, France, and NASA's Astrophysical Data System.

\section{REFERENCES}

Aikawa, T. 2010, A\&A, 514, A45

Arkhipova, V. P., Ikonnikova, N. P., \& Komissarova, G. V. 2010, AstL, 36, 269 Arkhipova, V. P., Ikonnikova, N. P., \& Komissarova, G. V. 2011, AstL, 37, 635 Arkhipova, V. P., Ikonnikova, N. P., \& Noskova, R. I. 1993, AstL, 19, 169 Arkhipova, V. P., Ikonnikova, N. P., Noskova, R. I., \& Sokol, G. V. 2000, AstL, 26, 609

Arkhipova, V. P., Noskova, R. I., Ikonnikova, N. P., \& Komissarova, G. V. 2003, AstL, 29, 480

Bakker, E. J., van Dishoeck, E. E., Waters, L. B. F. M., \& Schoenmaker, T. 1997, A\&A, 323, 469

Barnes, T. G. 2009, in Stellar Pulsation: Challenges for Theory and Observation, ed. J. A. Guzik \& P. A. Bradley (New York: AIP), 3

Barthès, D., Lèbre, A., Gillet, D., \& Mauron, N. 2000, A\&A, 359, 168

Beaulieu, J. P., Krockenberger, M., Sasselov, D. D., et al. 1997, A\&A, 321, L5 Blöcker, T. 1995, A\&A, 297, 755
Breger, M., Stich, J., Garrido, R., et al. 1993, A\&A, 271, 482

Cox, A. N. (ed.) 2000, Allen's Astrophysical Quantities (4th ed.; New York: Springer)

Fletcher, J. M., Harris, H. C., McClure, R. D., \& Scarfe, C. D. 1982, PASP, 94, 1017

Fokin, A. B., Lèbre, A., Le Coroller, H., \& Gillet, D. 2001, A\&A, 378, 546

Hrivnak, B. J. 1995, ApJ, 438, 341

Hrivnak, B. J. 1997, in IAU Symp. 180, Planetary Nebulae, ed. H. J. Habing \& H. J. G. L. M. Lamers (Dordrecht: Kluwer), 303

Hrivnak, B. J., \& Kwok, S. 1991, ApJ, 371, 631

Hrivnak, B. J., Kwok, S., \& Geballe, T. R. 1994, ApJ, 420, 783

Hrivnak, B. J., \& Lu, W. 2000, in IAU Symp. 177, The Carbon Star Phenomenon, ed. R. F. Wing (Dordrecht: Kluwer), 293

Hrivnak, B. J., Lu, W., Maupin, R. E., \& Spitzbart, B. D. 2010, ApJ, 709, 1042 (Paper I)

Hrivnak, B. J., Lu, W., Wefel, K. L., et al. 2011, ApJ, 734, 25

Hrivnak, B. J., Volk, K., Geballe, T. R., \& Kwok, S. 2008, in IAU Symp. 251, Organic Matter in Space, ed. S. Kwok \& S. A. Sanford (Cambridge: Cambridge Univ. Press), 213

Kiss, L. L., Derekas, A., Szabó, Gy. M., Bedding, T. R., \& Szabados, L. 2007, MNRAS, 375, 1338

Klochkova, V. G., Panchuk, V. E., \& Tavolganskaya, N. S. 2009, AstBu, 64, 155

Klochkova, V. G., Panchuk, V. E., \& Tavolganskaya, N. S. 2010, ARep, 54, 234

Lenz, P., \& Breger, M. 2005, CoAst, 146, 53

McLaughlin, D. B. 1941, ApJ, 94, 94

Nardetto, N., Gieren, W., Kervella, P., et al. 2009, A\&A, 502, 951

Omont, A., Loup, C., te Lintel Hekkert, P., Habing, H., \& Sivagnanam, P. 1993, A\&A, 267, 515

Ostlie, D. A., \& Carroll, B. W. 2007, An Introduction to Modern Stellar Astrophysics (2nd ed.; San Francisco, CA: Addison-Wesley)

Pollard, K. R., Cottrell, P. L., Kilmartin, P. M., \& Gilmore, A. C. 1996, MNRAS, 279, 949

Pollard, K. R., Cottrell, P. L., Lawson, W. A., Albrow, M. D., \& Tobin, W. 1997, MNRAS, 286, 1

Raskin, G., Van Winckel, H., Hensberge, H., et al. 2011, A\&A, 526, 69

Reddy, B. E., Lambert, D. L., Gonzalez, G., \& Yong, D. 2002, ApJ, 564,482

Scarfe, C. D. 2010, Obs, 130, 214

Scarfe, C. D., Batten, A. H., \& Fletcher, J. M. 1990, PDAO, 18, 21

Siodmiak, N., Meixner, M., Ueta, T., et al. 2008, ApJ, 677, 382

Steffen, M., Szczerba, R., \& Schönberner, D. 1998, A\&A, 337, 149

Udry, S., Mayor, M., \& Queloz, D. 1999, in ASP Conf. Ser. 185, Precise Stellar Radial Velocities, ed. J. B. Hearnshaw \& C. D. Scarfe (San Francisco, CA: ASP), 367

Ueta, T., Meixner, M., \& Bobrowsky, M. 2000, ApJ, 528, 861

Upgren, A. R., Sperauskas, J., \& Boyle, R. P. 2002, BaltA, 11, 91

Van Winckel, H., Lloyd Evans, T., Briquet, M., et al. 2009, A\&A, 505, 1221

Van Winckel, H., \& Reyniers, M. 2000, A\&A, 354, 135

Volk, K., Hrivnak, B. J., Matsuura, M., et al. 2011, ApJ, 735, 127

Volk, K., Kwok, S., \& Hrivnak, B. J. 1999, ApJL, 516, L99

Volk, K., Kwok, S., Hrivnak, B. J., \& Szczerba, R. 2002, ApJ, 567, 412

Wahlgren, G. M. 1993, in ASP Conf. Ser. 45, Luminous High-Latitude Stars, ed. D. D. Sasselov (San Francisco, CA: ASP), 270

Začs, L., Klochkova, V. G., \& Panchuk, V. E. 1995, MNRAS, 275, 764

Začs, L., Sperauskas, J., Musaev, F. A., et al. 2009, ApJL, 695, L203 


\title{
ERRATUM: “STUDIES OF VARIABILITY IN PROTO-PLANETARY NEBULAE. II. LIGHT AND VELOCITY CURVE ANALYSES OF IRAS 22272+5435 AND 22223+4327” (2013, ApJ, 766, 116)
}

\author{
Bruce J. Hrivnak ${ }^{1,6}$, WenXian Lu ${ }^{1}$, Julius Sperauskas ${ }^{2}$, Hans Van Winckel ${ }^{3}$, David Bohlender ${ }^{4}$, and Laimons Začs ${ }^{2,5}$ \\ ${ }^{1}$ Department of Physics and Astronomy, Valparaiso University, Valparaiso, IN 46383, USA; bruce.hrivnak@valpo.edu, wen.lu@valpo.edu \\ 2 Vilnius University Observatory, Ciurlionio 29 Vilnius 2009, Lithuania; julius.sperauskas@ff.vu.lt \\ ${ }^{3}$ Instituut voor Sterrenkunde, K.U. Leuven University, 3001 Leuven (Heverlee), Belgium; Hans.VanWinckel@ster.kuleuven.be \\ ${ }^{4}$ Dominion Astrophysical Observatory, Herzberg Institute of Astrophysics, National Research Council of Canada, 5071 West Saanich Road, \\ Victoria, BC V9E 2E7, Canada; David.Bohlender@nrc-cnrc.gc.ca \\ ${ }^{5}$ Faculty of Physics and Mathematics, University of Latvia, Raina bulvaris 19, LV-1586 Riga, Latvia; zacs@ latnet.lv \\ Received 2013 August 21; published 2013 October 17
}

At the time of the publication of this paper, we were not aware of the recent photometric study of IRAS $22272+5435$ by Arkhipova et al. (2009) and thus did not cite it in our study. They combined new observations from 2000-2008 with their previous data and carried out a period analysis of their entire data set. They also find two closely spaced periods that beat against each other to produce the modulated light curves. Their values are close but not identical to ours. They find periods of 128 and 131 days, and a third weaker one of 125 days; from their 2000-2008 data alone they find a dominant period of 128 days. These can be compared with our results, based on a larger data set of our 1994-2011 and their 1991-1999 measurements combined, of a dominant period of 131 days and a secondary period of 125 days. We did not find a period of 128 days, even when we analyzed our 2002-2011 data alone.

They also make a comparison between the light curves and radial velocity curves, in their case using only the 2006 radial velocity curves of Začs et al. (2009). Contrary to the earlier statement by Hrivnak \& Lu (2000), based on a small data set, and the conclusions of Arkhipova et al. (2009), the star is not brightest at its average size and expanding, but is actually brightest when smallest and faintest when largest, as seen in our Figure 7 and also in their Figure 4.

\section{REFERENCES}

Arkhipova, V. P., Ikonnikova, N. P., \& Komissarova, G. V. 2009, Peremennye Zvezdy (Variable Stars), 29, No. 1

Hrivnak, B. J., \& Lu, W. 2000, in IAU Symp. 177, The Carbon Star Phenomenon, ed. R. F. Wing (Dordrecht: Kluwer), 293

Začs, L., Sperauskas, J., Musaev, F. A., et al. 2009, ApJL, 695, L203

\footnotetext{
6 Guest investigator, Dominion Astrophysical Observatory, Herzberg Institute of Astrophysics, National Research Council of Canada.
} 